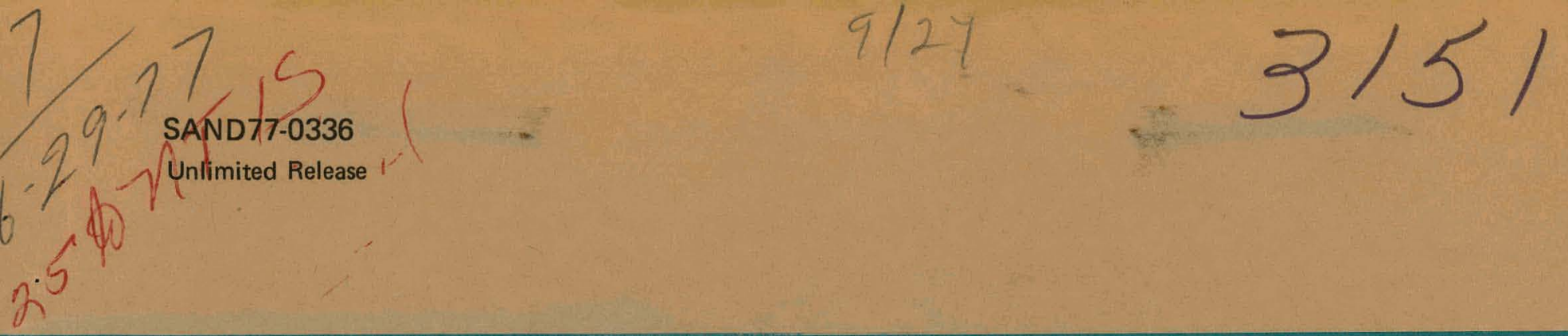

\title{
Status Report on the Development of a Prompt Fission Neutron Uranium Borehole Logging Technique
}

Gerald W. Smith

Prepared by Sandia Laboratories, Albuquerque, New Mexico 87115

and Livermore, California 94550 for the United States Energy Research

and Development Administration under Contract AT (29-1)-789

Printed May 1977

\section{[i] Sandia Laboratories energy report}
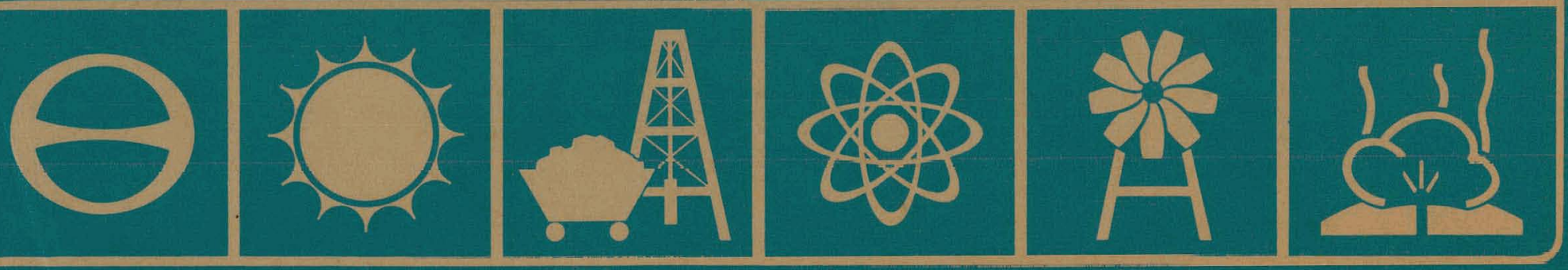


\section{DISCLAIMER}

This report was prepared as an account of work sponsored by an agency of the United States Government. Neither the United States Government nor any agency Thereof, nor any of their employees, makes any warranty, express or implied, or assumes any legal liability or responsibility for the accuracy, completeness, or usefulness of any information, apparatus, product, or process disclosed, or represents that its use would not infringe privately owned rights. Reference herein to any specific commercial product, process, or service by trade name, trademark, manufacturer, or otherwise does not necessarily constitute or imply its endorsement, recommendation, or favoring by the United States Government or any agency thereof. The views and opinions of authors expressed herein do not necessarily state or reflect those of the United States Government or any agency thereof. 


\section{DISCLAIMER}

Portions of this document may be illegible in electronic image products. Images are produced from the best available original document. 
Issued by Sandia Laboratories, operated for the United States Energy Research and Development Administration by Sandia Corporation.

\section{NOTICE}

This report was prepared as an account of work sponsored by the United States Government. Neither the United States nor the United States Energy Research and Development Administration, nor any of their employees, nor any of their contractors, subcontractors, or their employees, makes any warranty, express or implied, or assumes any legal liability or responsibility for the accuracy, completeness or usefulness of any information, apparatus, product or process disclosed, or represents that its use would not infringe privately owned rights. 
SAND 77-0336

Unlimited Release

STATUS REPORT ON THE DEVELOPMENT OF A PROMPT FISSION NEUTRON URANIUM BOREHOLE LOGGING TECHNIQUE

Gerald $W$. Smith

Optoelectronics Division 2542

Sandia Laboratories

Albuquerque, New Mexico 87115

ABSTRACT

The prompt fission neutron (PFN) method of direct uranium measurement was studied. The PFN uranium logging technique measures the enhanced epithermal neutron population created by the prompt thermal fission of ${ }^{235} \mathrm{U}$ to assay uranium mineralization around a borehole. This neutron population exists for several hundred microseconds after a pulsed neutron source produces a burst of high energy (14 MeV) neutrons. A feasibility study established the basic relationship between the uranium concentration and the enhanced epithermal neutron count, and defined the major measurement perturbing factors. Following the feasibility study, development of a PFN prototype field probe was undertaken. A laboratory type neutron generator, the Controlatron, was modified for use in the probe. Field evaluation of the prototype system began in January 1976. Comparisons of neutron logs and natural gamma logs taken during this evaluation period clearly define many disequilibrium conditions as verified by ore grade estimates from core samples. The feasibility of the PFN logging technique to detect uranium in-situ has now been demonstrated.
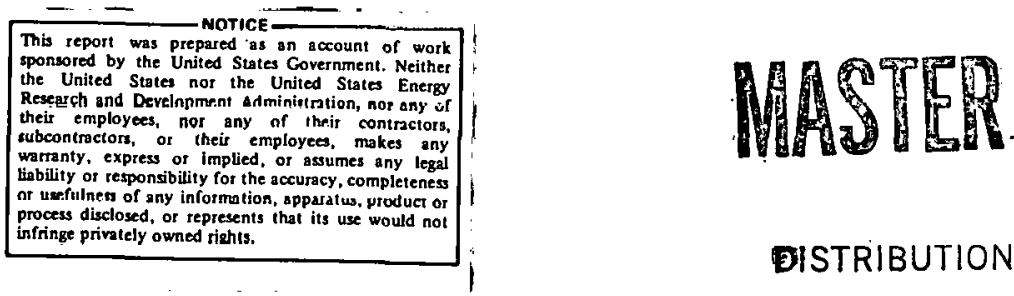
This document describes work done by Sandia Laboratories to evaluate the Prompt Fission Neutron (PFN) method of uranium logging. Included are descriptions of the feasibility study, the development and field evaluation of a prototype probe using the PFN technique and the logs taken during the field evaluation.

This is an active, ongoing program that began in October 1974, however, the activities covered by this document establish the development status as of approximately July 1 , 1976. since that time, significant advances in the use of the PFN logging technique have occurred, some of which have already been included in the field hardware.

The Sandia personnel who have participated in the PFN logging technique development program, beginning with the feasibility study and including the development and field evaluation of the prototype PFN probe up to July 1, 1976 are listed alphabetically. H. M. Bivens, E. L. Jacobs, D. H. Jensen, J. H. Renken, L. G. Rice, E. F. Richardson, G. W. Smith, and N. D. Wyatt.

The neutron generator used in the prototype PFN probe was developed for this logging application by the General Electric Neutron Devices Department, St. Petersburg, Florida. The GE personnel who participated in the development are listed alphabetically. B. R. Blitch, G. L. Heim, A. W. Pearson, F. D. Thibideau, and W. Washington. 
The activities reported herein were the result of a team effort by the Uranium Logging Project group consisting of H. M. Bivens, E. L. Jacobs, D. H. Jensen, L. G. Rice, and G. W. Smith.

The author wishes to express his sincere appreciation to R. W. Barnard, H. M. Bivens, and D. H. Jensen for valuable discussions and advice. 
Foreword

Acknowledgements

Introduction

PFN Logging

7

Theory

Feasibility study

Theoretical

7

Experimental

7

9

Priululype Fiobe Developmont

Neutron Generator

Reservoir Control

Source Neutron Detector

Epithermal Neutron Detector

Converter

Probe Housing

Up-Hole Instrumentation

Data System

Logging Vehicle

Data Interpretation

Conclusions

References

Figures 


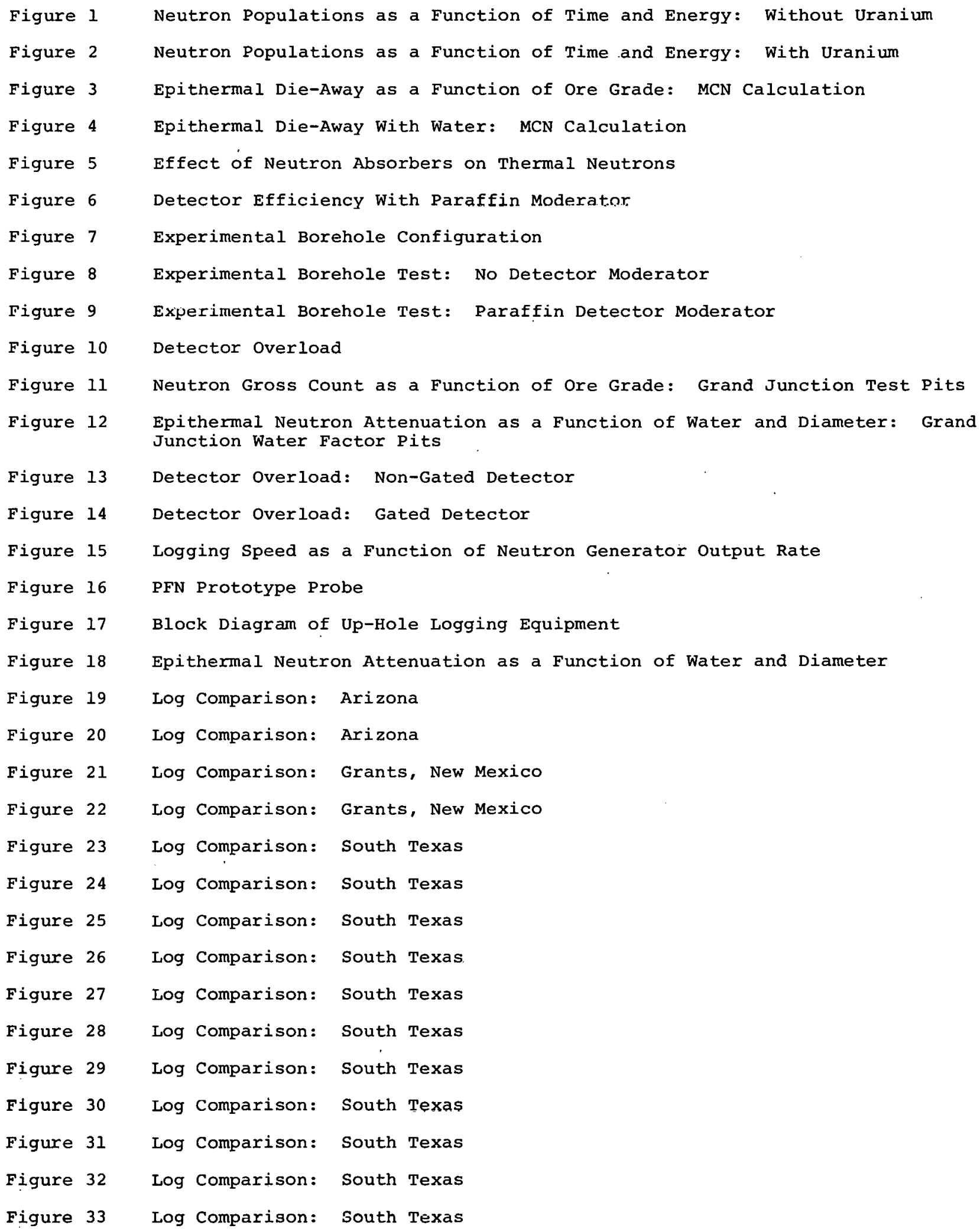


STATUS REPORT ON THE DEVELOPMENT OF A PROMPT FISSION NEUTRON

URANIUM BOREHOLE LOGGING TECHNIQUE

Introduction

For years, the primary method of uranium logging has been the detection of gamma radiation from daughter products of the natural decay of uranium. This method is accurate if the deposit is in equilibrium, i.e., the gamma producing daughter products are being created at the same rate they decay. This equilibrium condition depends on the parent uranium maintaining a stable posture in a formation for approximately $10^{6}$ years. Unfortunately, the uranium in a deposit can be preferentially dissolved in the ground water, transported to another location, and precipitated into another formation. This new deposit requires approximately $10^{6}$ years to reestablish the equilibrium condition since the daughter products, being chemically more stable, remain in the original deposit. This process is called leaching, and the expanding search for uranium has shown it to be increasingly prevalent. A natural gamma $\mathrm{log}$ of a formation in disequilibrium will clearly produce an incorrect estimate of the uranium in the deposit. In addition, natural gamma radiation from elements such as ${ }^{40} \mathrm{~K}$ and ${ }^{232} \mathrm{Th}$ may also obscure the true uranium concentration inferred from natural gamma logs.

In order to avoid both the problems of disequilibrium and interfering natural gamma radiation from elements not related to uranium, direct measurement of uranium by commercial prospectors has of necessity been done by analysis of core samples taken from exploration holes. However, the recovery and analysis of core samples is both expensive and timeconsuming and often is used only to confirm the presence of uranium indicated by significant natural qamma radiation. It is likely that recently deposited uranium will remain undetected because of low natural gamma radiation from daughter products that have yet to reach equilibrium.

In the hope of reducing this delay and expense, proposals for alternative methods of direct detection of uranium were made as early as 1961. (1) In recent years, two methods of direct uranium measurement by active interrogation with neutrons have been studied theoretically and experimentally. $(2 i s)$ Both methods detect neutron populations that result trom uranium fission produced by neutrons generated by the interrogation instrumentation.

The DFN (delayed fission neutron) technique measures the long term neutron population (delayed fission neutrons) produced by fission fragments decaying to stable states. The bulk of this neutron population occurs over a period of several seconds fulluwing fission uf the uranium atom. Two variations of the DFN technique have undergone considerable study and field evaluation, one using a pulsed neutron generator ${ }^{(3)}$ and one using an isotopic neutron source. (4)

The PFN (prompt fission neitron) technlque uses the neutron population created by. Lhe prompt thermal fission of ${ }^{235} \mathrm{U}$. This neutron population exists for several hundred microseconds after a burst of high energy neutrons from a pulsed neutron source. As of October 1974, the PFN technique had had little experimental study to support the theoretical predictions of Czubek. (2) 
In October 1974, under contract with the U. S. Energy Research and Development Administration (ERDA) field office in Grand Junction, Colorado, Sandia Laboratories began the first phase of a project to evaluate the PFN technique. This report describes the results of that study and the status of the program as of July 1, 1976.

PFN Logging

Theory

A burst of $14 \mathrm{MeV}$ neutrons generated by the $(d, t)$ reaction in a neutron generator tube will diffuse into the surrounding medium and produce several neutron populations as a function of time and energy. The high energy (14. MeV) neutron population quickly loses energy through collisions with nuclei in the formation, consequently the epithermal neutron population (energies between $\mathrm{l} \mathrm{KeV}$ and $0.5 \mathrm{eV}$ ) first increases and then decays as collisions, primarily with hydrogen, further reduce the energy of the neutrons. As the neutrons continue to lose energy and pass through the epithermal energy region, the thermal neutron population (energies below $0.5 \mathrm{ev}$ ) first increases and then dies away. The thermal neutrons are lost either through diffusion or absorption by nuclei in the formation. The thermal neutron population die-away time is predominantly governed by the macroscopic absorption cross section, of the formation. Figure 1 illustrates the neutron populations as a function of time that exist in a zone with no uranium mineralization.

The presence of uranium in the formation modifies the epithermal neutron population and its time distribution. This epithermal neutron population enhancement results from thermal fissioning of the ${ }^{235} \mathrm{U}$. The fission neutrons, originating with an average energy of 2 MeV, lose energy through collisions as the source neutrons did, creating a new population of epithermal neutrons. Figure 2 illustrates the neutron populations as a function of time that occur in the presence of uranium mineralization, with the enhanced epithermal neutron population shown in crosshatch. The decay rate (die-away time) of the epithermal neutron population, resulting from the presence of uranium, is the same as the decay rate of the thermal neutrons.

The enhanced epithermal neutron population is directly proportional to the amount of uranium mineralization in the zone of investigation if all other. formation parameters, such as absorption cross section, bulk density, porosity, and water saturation, remain constant. It is the enhanced epithermal neutron population that the PFN (prompt fission neutron) logging technique measures to assay uranium mineralization around the borehole.

\section{Feasibility Study}

The goal of the feasibility study was to establish the relationship between uranium ore grade and enhanced epithermal neutron die-away both theoretically and experimentally and to define the major perturbing factors affecting the measurement. These efforts began concurrently in October 1974 .

\section{Theoretical}

Analytically, the program began with the adaptation of an existing three-dimensional Monte Carlo neutron transport computer code $(\mathrm{MCN})^{5}$ which was originally developed at the 
Los Alamos Scientific Laboratory (LASL). The modified MCN code was used to model threedimensional variations of a simulated borehole and to determine the space, time, and energy distribution of neutrons resulting from a high energy ( $14 \mathrm{MeV}$ ) neutron burst. The parameters of primary interest, i.e., ore grade, geometry, borehole and formation water, bulk density, and impurity concentration, all affect the epithermal neutron die-away characteristics.

In order to improve measurement statistics without a corresponding increase in the $\mathrm{MCN}$ computer run time, some modifications were incorporated in the MCN code. Two of the most useful were thermal splitting and artificial ore enhancement.

Thermal splitting is a technique by which neutrons reaching thermal energies are split into several thermal neutrons with a corresponding reduction in weighting factor. Since the thermal neutron population produces the enhanced epithermal neutron population by ${ }^{235} \mathrm{U}$ fission, an improvement in epithermal neutron statistics results without the necessity of generating increased numbers of $14 \mathrm{MeV}$ neutrons.

Artificial ore concentration enhancement increases the probability of a neutron collision with a fissionable nucleus. The neutron Monte Carlo calculation is performed using the true ore concentration, which correctly simulates the diffusion parameters; however, the artificially increased ore concentration increases the collision probability between a thermal neutron and ${ }^{235} \mathrm{U}$. The effective weighting factors of any fission neutrons resulting from a collision are reduced by an amount proportional to the ore enhancement.

The use of these code modifications significantly reduced the statistical fluctuation of epithermal neutron die-away calculations. Figure 3, which is based on a simplified geometry and a homogeneous matrix surrounding the borehole, clearly reveals the dependence of the enhanced epithermal neutron population on ore grade. Unfortunately, calculations for concentrations of $\mathrm{U}_{3} \mathrm{O}_{8}$ below 0.1 percent using $\mathrm{MCN}$, even with the improvements described, become increasingly unreliable because of statistical scatter. Although this shortcoming of the MCN code restricted the scope of its analytical use, it was used to analyze epithermal neutron response variations produced by several parameters, in addition to ore concentration

Figure 4 shows the time spread of the epithermal neutron population resulting from $14 \mathrm{MeV}$ neutron bursts in a homogeneous barren zone as a function of formation water content. The die-away time of the epithermal neutron population resulting from source neutrons will determine the start time of the measurement window in which the enhanced epithermal neutron measurement can begin without interference from source epithermal neutrons. From this result, it is clear that the measurement of enhanced epithermal neutrons in very dry formations must not begin prior to $200 \mu \mathrm{s}$ following the source neutron burst.

An analysis of thermal neutron population reduction due to elements with high thermal neutron absorption cross sections is shown in Fiqure 5 . The typical concentration of these elements that can be expected in sandstone is low enough to generally disregard this effect in normal deposits. Since the probability of a significant effect from thermal neutron absorbers is small, no effort was made in the early instrumentation packages to monitor for this effect.

Early in the feasibility study, a ${ }^{3}$ He Proportional counting (PC) tube was selected as the epithermal neutron detector because of its operational simplicity and insensitivity to gamma radiation. The neutron detection sensitivity is high ( $\approx 100 \%$ ) for thermal neutrons but decreases as the neutron energy increases. Since the ${ }^{3} \mathrm{He}$ PC tube is intended to be an epithermal neutron detector, the high thermal neutron sensitivity is effectively eliminated by a thin wrap of cadmium which absorbs thermal neutrons before they reach the ${ }^{3} \mathrm{He}$ PC tube. 
The decreasing neutron sensitivity to the more energetic neutrons results in a very low effective detection probability for the enhanced epithermal neutron population (about 108). The possibility of improving the detector efficiency for epithermal neutrons by surrounding the PC tube with neutron moderating material such as paraffin was investigated by MCN analysis. The results of this analysis suggest significant improvement in detector efficiency can be realized as shown in Figure 6. Unfortunately, another problem, detector overload, is aggravated by the paraffin moderator. Detector overload is examined in the Experimental discussion.

While the MCN transport code was being used for this type of analysis, the experimental program began investigating some of the theoretical predictions.

\section{Experimental}

The first experimental verification of the proposed PFN technique in a borehole-type environment was attempted in four simulators made from 55-gallon oil drums modified by mounting a concentric, $11 \mathrm{~cm}$ diameter, thin walled aluminum tube in the center along the drum axis. The drum annulus was filled with the material to be tested, such as wet and dry ore containing 0.2 percent $\mathrm{U}_{3} \mathrm{O}_{8}$ and wet and dry sand barren of $\mathrm{U}_{3} \mathrm{O}_{8}$. Each of the four models represented a homogeneous condition for which a distinguishable epithermal neutron time dependent response was expected.

The first "probe" was an assembly of available laboratory hardware, composed of a high energy pulsed neutron source and a neutron detector in close proximity. The neutron source was a laboratory type unit. with portable tube transformer assembly (TTA) and rack mounted power supplies and controls. It had a maximum output of $2.5 \times 10^{7}$ neutrons per pulse and a capability of one pulse every two seconds. The neutron detector consisted of a ${ }^{3} \mathrm{He}$ proportional counting tube $50 \mathrm{~mm}$ in diameter with a $254 \mathrm{~mm}$ active length under $10^{6} \mathrm{~Pa}$ of pressure; it was wrapped with a $0.76 \mathrm{~mm}$ thickness of cadmium and included a small electronic package for signal conditioning. Power supplies and detector controls were rack mounted.

Although the "probe" hardware was somewhat limited, it was nevertheless adequate to perform this first qualitative experiment. The data handling system, however, was not as easily assembled from available hardware. It was believed that in addition to the gross count of the epithermal neutron population relating directly to the grade of the ore, significant information concerning measurement perturbing effects could be derived from the epithermal neutron die-away characteristics. The requirements for time resolution of the epithermal signal neutron population considerably complicated the measurement. Not only was the time resolution of the epithermal neutron population more complex, but an appropriate storage method for each data point had to be included. The measurement system chosen to meet these requirements included a multiscaler/multichannel analyzer $(6)$ and tape drive $(7)$ with appropriate interface. For multiscaler operation, 50 microsecond time windows were selected as a compromise between adequate counting statistics and resolution for die-away analybie.

The first qualitative tests were designed to verify that significantly different epithermal neutron lifetime characteristics would result from measurements in the four borehole models previously described. From estimates derived from both Czubek's (2) work and MCN transport analysis, it was determined that $10^{9}$ source neutrons per datum point would be used to produce a series of measurements for the four drum models.

These tests produced the unexpected result that no significant epithermal neutron 
population change occurred for any borehole model. The reason for this result was shown to be the limited material within the small dimensions of the drum models which prevented sufficient thermalization of the high energy source neutrons by allowing significant numbers to escape before fission reactions with the ${ }^{235} \mathrm{U}$ occurred. Clearly, a more realistic borehole model was required for even the most rudimentary verification testing of the $\mathrm{PFN}$ technique.

A suitable location was selected near the mobile laboratory which was being outfitted for tests at the ERDA Grand Junction test pits. The construction of the test pit was geometrically similar to the Grand Junction pits, but was designed only for short term, qualitative tests. The ore used in this construction was dried such that less than $I$ percent $\mathrm{H}_{2} \mathrm{O}$ by weight remained. A low moisture condition was considered to be the simplest to control and thereby maintain consistency. Figure 7 illustrates the configuration of the Sandia test pit.

Tests in the new pit produced encouraging results. Significant enhanced epithermal neutron returns were detected with the probe in the "ore zone", while no enhanced epithermai neutrons occurred with the "probe" below the ore zone in barren ground. This measurement series used the same laboratory neutron generator and proportional counter detector used in the drum model tests. A typical result of these measurements can be seen in Figure 8 which shows an enhanced epithermal neutron population in the ore zone and no enhanced epithermal returns in the barren zone.

As a result of trying to improve detector efficiency by including a paraffin moderator annulus between the cadmium wrap and the ${ }^{3} \mathrm{He}$ PC tube, the problem of overloading the ${ }^{3} \mathrm{He}$ PC tube became severe. As shown by Figure 9, a significant loss of epithermal neutron counts occurs during the early die-away period. Much of the early epithermal neutron population is not counted due to a change in detector characteristics produced by the capture of a large number of high energy source neutrons. Although the detection probability of a $14 \mathrm{MeV}$ neutron is extremely small, their presence in great abundance (> $10^{7}$ per pulse) results in the capture of sufficient numbers to temporarily disable the ${ }^{3} \mathrm{He}$ PC tube. The presence of paraffin further exaggerates the overload effect by reducing the energy of some of the $14 \mathrm{MeV}$ source neutrons through collisions with hydrogen nuclei, resulting in a higher detection probability. During this overload period, the measurement of the epithermal neutron population is in considerable error, which leads to poor estimates of both total count and die-away characteristics.

As seen by a comparison of Figures 8 and 9, the paraffin moderator does produce an improvement in detector efficiency after recovery, as predicted from the MCN analysis shown in Figure 6 .

Several possibilities were considered for eliminating the overload problem. The possibility of alternative detection methods was considered, but the test schedule did not permit the evaluation of alternatives. An alternative detector is still a subject under investigation and may in the future provide significant advantages.

Several methods of improving the overload recovery of the ${ }^{3} \mathrm{He}$ PC detector were considered, including modification of the initial probe configuration. The source and the detector spacing as well as the separation material were tested to define the optimum geometry. Figure 10 illustrates the results of these tests. While none of the geometric combinations provided sufficient improvement, an air filled separation of $13 \mathrm{~cm}$ between source and detector produced the most rapid recovery from overload. 
Since an adequate answer was not found by changing the geometry, the possibility of significant improvement resulting from bias voltage gating was considered. Preliminary tests indicated a reduction in overload and faster recovery time resulted from reducing the detector bias to a point just below the charge multiplication potential. However, the schedule for tests at Grand Junction did not permit a modification of the detector electronics prior to the equipment move for the first series of tests at the ERDA pits. The first experiments at Grand Junction were done with a constant voltage biased detector.

In the belief the ERDA test pits at Grand Junction are characterized by well known geometry, ore grade, density, and water content, they were considered to be the standard to which tests results would be compared. Of particular interest in this experiment was: (1) the nature of the relationship between enhanced epithermal neutron population and ore grade, and (2) the relation between the enhanced epithermal neutron die-away characteristics and perturbing effects due to formation parameters such as bulk density and formation moisture content.

Proportionality between the enhanced epithermal neutron gross count and the ore grade was clearly evident. Figure 11 shows the enhanced epithermal neutron gross count as a function of ore grade for $10^{9}$ source neutrons. The enhanced epithermal neutron population die-away characteristics measured in several different test pits, exhibited variations that were not simply related to the formation parameters, and a satisfactory relationship could not be established at this point.

In order to analyze the time dependent characteristics of the signal neutrons, several closed form mathematical models were compared by least squares fit ${ }^{(\theta)}$ to a special series of data which consisted of ten independent measurements at the same position in the same test pit (U-1). An exponential, weighted such that measurement time intervals containing many counts dominated the least squares fit, produced the best agreement with the data. By using this model, the decay constant of the exponential can be used to describe the enhanced epithermal neutron population die-away. The inverse of the decay constant is the mean lifetime of this population. The relation between this mean lifetime and formation parameters is still under investigation.

Another measurement of significance produced during this series indicated the effect that water in the borehole has on epithermal neutron gross count. Figure 12 shows this effect as a function of borehole diameter.

Since the detector was operated at fixed bias voltage, an effort was made to betit.r. define the relation between source neutrons per pulse and signal neutrons per measurement. The neutron generator had the capability of lower neutron output per pulse by reducing the accelerating potential. By appropriate adjustment, levels as low as $5 \times 10^{5}$ neutrons per pulse could be achieved. Figure 13 shows the effective detector response as a function of neutron generator output per pulse.

Prior to a second series of tests at Grand Junction, a successful detector bias voltaqe gating circuit was developed that provided significantly improved operation in the presence of high energy source neutron bursts. The circuit produces approximately a 30 percent reduction in PC tube voltage bias, to a level below avalanche potential, for a period of $50 \mu \mathrm{s}$. During this interval, the neutron source is pulsed, but the free charge produced in the PC detector is not multiplied. Without charge multiplication, less free charge is created and less time is required to collect it. Figure 14 shows the improvement in detector recovery from overload produced by bias voltage gating. Detector counts in Figure 13 and 
Figure 14 are normalized to the same detector count.

The experimental program demonstrated conclusively that the PFN logging technique could be successfully used to assay uranium ore directly. However, the question of how accurately this measurement can be made has only been partially answered.

Prototype Probe Development

Following the successful completion of the feasibility study, program emphasis yuickly shifted to the design and development of a logging probe to further study the PFN logging method under field conditions. The design definition of such a system began in August 1975 , with a projected field operational date of January 1, 1976.

The transition from an assortment of laboratury instrumerls capable of some measurements under controlled conditions in the ERDA tesls pits to a self-contained instrumentation system capable of logging under field conditions was not trivial in the time scale involved. Development of much of the hardware necessary for such a system might well be considered straightforward, but one component, the neutron generator, required considerable develupillelit effort.

\section{Neutron Generator}

The pulsed neutron generator adapted for the probe consisted of several individual components: (1) neutron tube and high voltage transformer in one assembly for high voltage breakdown protection, (2) power supply and pulse forming network for electrical pulse drive, and (3) reservoir control to maintain the correct gas pressure in the neutron tube since it uses a cold cathode discharge ion source. For this logging configuration all of these components must be compatible with a probe housing with an inside diameter of $6.35 \mathrm{~cm}$.

Two parameters defined during the feasibility study determined the output requirements of a neutron generator that could be used as a PFN logging yenerator. First, the source neutron levei of $10^{\prime}$ neutrons per datum point was found to be marginally adequate for medium grade ore concentration $\left(\sim 0.28 \quad \mathrm{U}_{3} \mathrm{O}_{8}\right)$. Since low grade ore deposits in the order or 0.05 percent $\mathrm{U}_{3} \mathrm{O}_{8}$ are likely to be encountered in field exploration, a source neutron total per datum point of at least $5 \times 10^{9}$ would be required. In the logging mode, at least one datum point every $1 \overline{5} \mathrm{~cm}$ is desired in order to adequately define the deposit. These two requirements are related to logging rate and neutron generator output rate, shown in Figure 15. For the desired minimum of $5 \times 10^{9}$ source neutrons per measurement and one measurement every $15 \mathrm{~cm}$, a continuous logging rate of $1.83 \mathrm{~m}$ per minute would place a requirement of $10^{j}$ neutrons per second on the logging neutron generator. In addition to the neutron source output rate of at least $10^{9}$ neutrons per second, the neutron output per pulse, and hence the pulse rate. must be determined by a compromise between two conflicting requirements.

Detector "overload" can best be reduced by operating at very low pulse output and high pulse rates since detector bias gating can reduce but not eliminate the overload susceptibility of the ${ }^{3} \mathrm{He}$ PC detector. For a true neutron output rate (neutron per second) the efficiency of the generator increases as the neutron output per pulse increases.

Maximum generator pulse rate is limited by the time required to complete the measurement initiated by the neutron burst. The optimum measurement interval is a variable which depends on the ore grade and formation factors, particularly formation water content. The longest measurement interval encountered during the feasibility study approached $2000 \mu s$. 
In this case the measurement interval would permit a pulse rate of 500 pulses per second (pps) and a pulse output of $2 \times 10^{6}$ neutrons per pulse. At this level, there is no significant detector overload effect.

Another factor, related to the data system must be considered. A 256 channel multiscaler requires $12.8 \mathrm{~ms}$ to sweep at $50 \mathrm{\mu s}$ per channel. When the sweep begins, coincident to a neutron generator burst, the total $12.8 \mathrm{~ms}$ sweep must be completed before the multiscaler can accept data for the next measurement. However, since only 2 ms at most is required to make a measurement, most of the 256 channels are unused. A standard module can modify the sweep to only 64 total channels and reduce the sweep time to $3.2 \mathrm{~ms}$. The measurement interval would permit a maximum pulse rate of 300 pps and a generator pulse output of $3.2 \times 10^{6}$ neutrons, a level still essentially free from detector overload.

The minimum neutron generator pulse rate can be determined by the detector overload threshold. From Figure 14, the overload effect produces about a 10 percent reduction in detector sensitivity at an output of $5 \times 10^{6}$ neutrons per pulse, which corresponds to an operational pulse rate of 200 .pps.

Reliability and longevity are important characteristics for instrumentation expected to operate at remote exploration locations. The improved reliability resulting from 200 pps operation outweighted the slightly improved detector overload characteristics that would result with 300 pps operation.

The operational life of a neutron generator must be sufficiently long to allow several hours of total logging time and at least 15 minutes of continuous operation at the logging output level. A logging neutron generator operating at 200 pps approaches $10^{6}$ operations in about 1.4 hours. At a nominal logging rate of $1.83 \mathrm{~m}$ per minute, a total of only $154 \mathrm{~m}$ could be logged in 1.4 hours time, which would place severe field operational restrictions on the system. Fourteen hours of neutron generator operational life expectancy would be acceptable, for this development application; therefore, $10^{7}$ total operations was selected as the minimum desirable neutron generator life.

Since the epithermal neutrons detected during a logging operation will depend on, in addition to ore grade, the rate at which high energy source neutrons are produced by the neutron generator, an accurate source neutron monitor is required to normalize the measurement to a known quantity of source neutrons. In order to avoid measurement error due to interfelence from the external borehole environment, a source neutron monitor should be small with low sensitivity, which means it must be placed at a location in very close proximity to the neutron source. This arrangement of source neutron monitor and neutron target can only occur if the neutron tube is operated in the grounded target (neutron source) mode. Grounded target operation means the neutron tube ion source and ion source drive must be operated at high potential. In this application this is an operational mode that is more complex and difficult than operating the neutron tube with the target at high potential.

All of these factors define a set of desirable operational specifications for a proposed neutron generator which can be used for field evaluation of the PFN logging technique. In summary, the neutron generator must not have a diameter larger than $6 \mathrm{~cm}$, with an output rate capability of at least $10^{9}$ neutrons per second at a pulse rate of 200 pps. The pulse half width can be nominally 10 to $20 \mu \mathrm{s}$. The target should be at ground potential with ready acceso for a small, high energy (14 MeV) neutron detector. The operational life expectancy of the generator should be at least $10^{7}$ total operations. 
In August 1975, no such neutron generator was available either commercially or existed within the ERDA weapons complex. However, the neutron generator used for the feasibility experiments demonstrated some of the desired operating characteristics. It had been operated successfully, during its early development, in life tests of several million operations, and the individual pulse output could easily meet the $5 \times 10^{6}$ neutron level. The real question that required answering was whether an output rate of $10^{9}$ neutrons per second could be sustained in stable operation at 200 pps. The prospects of quickly fielding a PFN logging probe could be realized only if this laboratory neutron generator could be adapted to meet these operational specifications.

This neutron generator, called a Controlatron, is manufactured by the General Electric Neutron Devices Department (GEND), St. Petersburg, Florida. GEND is an ERDA integrated contractor which has production responsibility for neutron generators and which provides support to Sandia for neutron tube and neutron generator development. The Controlatron neutron generator had originally been developed by GE in the early 1960's for a lunar exploration application by NASA. Although never used for that purpose, the controlatron has been extensively used in the ERDA weapons complex as a stable neutron source for tester calibration. The laboratory controlatron uses a type $26 \mathrm{~A}$ neutron tube in which the ion beam target (neutron source) is located at the end of a $15 \mathrm{~cm}$ drift tube. It is operated in the grounded target mode to provide easy and close access to the neutron source. It was this type of Controlatron that was used during the feasibility experimental program. Another neutron tube, the $26 \mathrm{E}$, came under consideration for this application. The 26E is a modified $26 \mathrm{~A}$ tube from which the drift tube has been removed. The $26 \mathrm{E}$ tube has been operated with the ion beam target at dc high potential, with the ion source pulsed at rates as high as 5000 pulses per second, and with an output rate of approximately $10^{6}$ neutrons per second.

Early discussions between Sandia and GEND engineers resulted in a consensus that the Controlatron neutron generator could be modified for use as a PFN logging probe neutron generator in a four month period, provided some of the specifications were changed.

The first of the specifications to be revised dealt with the grounded target operation. Excessive distributed capacitance associated with the $26 \mathrm{~A}$ Controlatron tube source arid permanent magnet design resulted in prohibitive displacement current energy loss from the pulse forming storage network. It was decided that the problems associated with grounded target operation could not be successfully dealt with in the required time frame. It was decided to use the $26 \mathrm{E}$ controlatron tube and operate the ion beam target at high potential. This compromise had serious implications with regard to the source neutron monitor and $i t s$ accuracy. This will be discussed later.

The Controlatron tube transformer assemblies (TTA) are normally operated by switching a pulse forming network (lumped constant transmission line) charged to approximately $6 \mathrm{kV}$ onto the high voltage transformer primary. Switch tubes, either gas or vacuum, used at these voltage levels were found to have a severely limited operational life expectancy at the high power level needed for the logging application. The only possible alternative appeared to be a solid state switching device (SCR) which has no inherent wear out mechanism but has a much lower voltage holdoff capability (approximately $1 \mathrm{kV}$ for reliable long term use). The neutron tube accelerating potential must remain constant. Therefore, a factor of six reduction in the transformer primary voltage required a corresponding increase in the turns ratio of the high voltage transformer.

The reduction of the pulse forming network operating voltage to $1 \mathrm{kV}$ provided an 
opportunity for an overall efficiency improvement. Had the pulse forming network operated at $6 \mathrm{kV}$, a voltage conversion in the probe would have been required to increase the voltage of the primary power transmitted to the probe on the logging cable.

With the prospect of $1 \mathrm{kV}$ operation of the pulse forming network, a closer examination was made of $4 \mathrm{HO}$ logging cable to determine its maximum voltage handing capability.

Iiterature checks had indicated the voltage rating to be $100 \mathrm{~V}$, but investigation of the electrical insulation properties suggested adequate reliability at $500 \mathrm{~V}$ operation. High voltage tests of $4 \mathrm{HO}$ cable verified that 500 vdc between one conductor and shield or between two conductors could be done reliably. This situation permitted primary power to be supplied to the probe at $500 \mathrm{Vdc}$, at which point it was increased to $1 \mathrm{kV}$ by a ringing choke voltage doubler. This method eliminated the losses of a high voltage converter as well as reducing the $I^{2} R$ losses that would occur with low voltage, high current primary power in the cable.

Some concern developed at GEND that transformer heating in the TTA at the full output operation of 200 pps would degrade the high voltage insulation to the point that a breakdown failure might occur. For this reason, the pulse rate was reduced to 100 pps, with a corresponding factor of two reduction in neutron output to $5 \times 10^{8}$ neutrons per second.

The neutron generator output rate decreased, in the first few minutes of operation, to approximately $2 \times 10^{\circ}$ neutrons per second, due to target depletion. Most of the field logging covered in this report was done at a neutron generator output level not greater than $2 \times 10^{8}$ neutrons per second.

The compromises in neutron generator specifications were made to maximize the probability of the successful development of a useable PFN prototype probe in a very short time frame. A more detailed discussion of the development of this logging neutron generator has been published. (9)

\section{Reservoir Control}

A gas filled neutron tube with a Penning cold cathode ion source, such as a controlatron 26E, requires precise gas pressure control for proper ion source operation and stable neutron output. The reservoir, a porous stainless steel cylinder containing powdered titanium deuteride, releases deuterium gas in the neutron tube when electrically heated.

The ion source is operated by the application of a $2.5 \mathrm{kV}$ pulse which creates an electric field in which the free charge is accelerated. The path of this free charge is transverse to a permanent magnetic field which lengthens the path, thus increasing the collision probability of the free charge and a gas molecule. At the proper gas pressure, ions are produced and accelerated to the target, producing neutrons.

In operation, the gas pressure is dynamically related to the generator pulse rate through the loss of ions in the neutron generation process. To maintain proper gas pressure and stable neutron output in this dynamic condition, an electronic feedback loop was developer to control the reservoir drive current. The reservoir control circuit detects changes in the integral of the source voltage drive pulse (a monotonic function of gas pressure), and modulates the pulse width of a $10 \mathrm{~A}$ peak current pulse train which heats the reservoir. Although sudden extreme changes in the pulse rate of the neutron generator produce gas pressure changes which require several seconds to stabilize due to the relatively slow thermal response of the reservoir, in steady state operation the reservoir control circuit provides stable neutron output even at high pulse rates. 
Source Neutron Detector

As previously mentioned, compromises in neutron generator specifications resulted in a neutron generator configuration in which the ion beall target (neutron source) was not accessible. Generator operation in the grounded ion source mode requires that bulky transformer components and insulation material be arranged in positions which isolate the target. This configuration considerably complicates source neutron monitoring.

In the grounded ion source configuration, the source neutron detector was located approximately $20 \mathrm{~cm}$ from the neutron source. At this distance, the detector sensitivity requirement could most easily and quickly be realized by using a scintillator-photomultiplier combination.

Ideally, the detector would measure only source neutrons generated within a solid angle defined by the geometry of the detector (right circular cylindrical plastic scintillator $5 \mathrm{~cm}$ diameter $\mathrm{x} 5 \mathrm{~cm}$ height) and the separation from the neutron source (20 cm). However, high energy source neutrons which are generated outside chis sulic ailde and ocatter intn the detertor as a function of borehole parameters create an error in the measurement of the source neutron output.

Also included in this photomultiplier-scintillator package is electronic circuitry to integrate the pulse by pulse neutron detector ontput, and to produce a pulse (data dump signal) when $10^{9}$ total source neutrons have been generaled. The data dump pulse instructs the data system on the surface to recurd the multiscaler data on magnetic tape and to begin the summation of neutron counts for the next datum point.

Although tests of this source monitor system indicated errors as great as 30 percent, the test schedule of the PFN prototypc probe delayed the development of a more accurate source neutron detector until July 1976. For that reason, the system described was used during the entire course of experimentation covered in this rcport.

\section{Epithermal Neutron Detector}

The neutron generator compromise configuration previously described had still another effect on the overall system. It was found during the feasibility study that the ${ }^{3} \mathrm{He} P C$ detector with bias voltage gating could operate with zero end spacing from a neutron source producing $5 \times 10^{\circ}$ neutrons per puise and suffei only minor overload effert.s. Zero end spacing produced improved measurement response because of the closer proximity to the signal producing region of the ore zone under interrogation by source neutrons. The logging neutron generator configuration forced a displacenent of 20 cil butwen lie neutron 60 urso and the end of the ${ }^{3} \mathrm{He}$ PC tube $(33 \mathrm{~cm}$ from PC tube center), resulting in a reduction in count level and resolution of the ore zone.

The epithermal detector used in the prototype probe was modified only slightly from the feasibility instrumentation. The same ${ }^{3} \mathrm{He}$ PC tube (50 mm diameter $\mathrm{x} 254 \mathrm{~mm}$ active length) was used with modified electronics for rapid response bias voltage gating control and signal conditioning.

\section{Converter}

The 4HO logging cable, which is the industry standard for uranium logging, has four conductors and an armored shield which is used for both signal and power ground return. Three of the conductors are required for signal transmission and probe control, leaving the 
fourth for the transmission of $500 \mathrm{Vdc}$ primary power to the probe.

In order to operate the probe, electrical power at various voltages is required. The neutron generator operates directly on the 500 vdc primary power, but all of the probe components require $12 \mathrm{Vdc}$ for electronics. In addition, a $10 \mathrm{~A}$ dc current source is required by the reservoir control package to produce the pulse width modulated drive to the neutron tube reservoir. Since there are no available conductors in the logging cable for the transmission of this additional electrical power to the probe, it must be converted from the 500 Vdc. A specially designed voltage converter was obtained for this purpose. The efficiency of this converter is relatively poor $;$ and special measures are required to dissipate the heat generated.

\section{Probe Housing}

The probe housing is made of stainless steel seamless pipe having a $70 \mathrm{~mm}$ OD and a $3 \mathrm{~mm}$ wall. The pipe is machined to accommodate a piston nose piece and has a welded cap for lifting strength at the top. The probe in its operational configuration is $70 \mathrm{~mm}$ in diameter, $3.36 \mathrm{~m}$ in length, and it weighs approximately $40 \mathrm{~kg}$.

The wall thickness of the tube and "0" ring seals for the two end pieces will withstand a maximum pressures of approximately $2 \times 10^{7} \mathrm{~Pa}$ and permit logging at depths to $1200 \mathrm{~m}$. The probe nose seal is a piston which is removed for inserting or removing the probe components. Figure 16 defines the probe assembly in block diagram form.

Three of the probe components, the TTA, reservoir control circuit, and the converter, generate significant quantities of heat and required particular care to insure adequate heat transfer to the probe housing. The best solution found to provide the necessary heat transfer was a dielectric fluid ${ }^{(10)}$ which fills the probe and provides convective heat flow between the probe housing and the heat producing components.

\section{Up-Hole Instrumentation}

The basic system required above ground to provide power to the probe, control its operation and record data is defined schematically in Figure 17 . The probe in full operation requires approximately 150 watts of primary power, which is supplied by a commercial 250 watt dc power supply. (11)

Probe control is provided by a trigger system which establishes the proper time sequencing between the downhole probe operation and the uphole data acquisition system. The trigger system provides variable pulse repetition rates for several operational and calibration requirements. Single shot operation is used to observe the neutron source voltage pulse shape to assure safe neutron tube operation. One pulse every five seconds is used for absolute neutron generator output calibration. One pps is used for warm-up operation, neutron tube reservoir control adjustment, and as the standby mode for the probe. Forty pps operation was used for borehole survey and for reservoir control adjustment to 100 pps operation. One hundred pps operation currently is the maximum rate of neutron tube operation for logging. Until more experience gained through life testing of the logging neutron generator indicates that 200 pps operation can be sustained, field use will be restricted to 100 pps operation in the interest of neutron generator reliability.

\section{Data System}

During the feasibility experimental program, a datum point was produced by pulsing a 
neutron generator fixed at some test position in the borehole of a test pit until the desired source neutron output (generally $10^{9}$ neutrons) had been reached. At that point, the time resolved epithermal neutron population accumulated and stored in the multiscaler memory was transferred by manual initiation to magnetic tape. The instrumentation was then moved to a new test position at which a new datum point was generated and the cycle repeated.

In a continuous logging mode, this data accumulation and storage cycle can be controlled automatically with some compromise in standard multiscaler operation. During data transfer from multiscaler memory to magnetic tape, the multiscaler data acquisition capability is disabled for approximately $0.5 \mathrm{~s}$. The measurement accumulation time for the datum point for a nominal $15 \mathrm{~cm}$ depth increment is approximately $4.9 \mathrm{~s}$. This multiscaler dead time will cause the first 10 percent of a nominal $4.9 \mathrm{~s}$ measurement interval to be lost, creating a measurement condition for which distortion of an ore zone could result, particularly at points of high contrast in ore grade.

The multiscaler manufacturer ${ }^{(6)}$ was contacted with regard to the possibility of a modification to eliminate this dead time error. In response, they developed a buffer memory module (12) to record the real time data, which is then transferred on command to the multiscaler, from which it can be recorded on magnetic tape. The buffer memory module can recover and record new data within a few microseconds following the data transfer to the multiscaler memory, eliminating dead time 1oss. Also incorporated in the buffer module is a modification which reduces the effective multiscaler memory from 256 channels to 64 channels and this reduces the total measurement time interval after each pulse from $12.8 \mathrm{~ms}$ to $3.2 \mathrm{~ms}$.

\section{Logging Vehicle}

In order to transport and operate this logging system in the field, a suitable logging vehicle is essential. For this purpose, a surplus ERDA logging vehicle was made available on a loan basis to Sandia from the Grand Junction, Colorado, field office. The vehicle came to Sandia equipped with racks, a $4 \mathrm{~kW}, 110 \mathrm{~V}, 60 \mathrm{~Hz}$ single phase, auxiliary power unit (APU), and an electric winch with $620 \mathrm{~m}$ of used 4 Ho logging cable. Relatively minor modifications were required to accommodate the data acquisition system, the power and control system, and maintenance and troubleshooting hardware.

The complete logging system was first successfully tested on December 19, 1975.9 The first field operations were conducted in the Grants Mineral Belt on January $15,1976$.

\section{Data Interpretation}

In January 1976, field evaluation of the PFN (Prompt Fission Neutron) logging technique using the prototype probe began near Grants, New Mexico. Subsequent field evaluation trips were made to exploration properties in Arizona, New Mexico, and Texas, during the next six month period ending in June 1976.

Field expeditions were restricted to sites at which the exploration companies agreed to furnish grade analysis of core samples as well as conventional natural gamma logs. The core information is used as the ore grade standard to which the neutron and gamma logs are compared. Such comparisons were expected to demonstrate the superiority of the neutron log in the presence of disequilibrium. Core information is also being used to assist in the development of corrections for measurement perturbing effects. 
During this first field evaluation period, the field data taken by the PFN system were limited to a measurement of the epithermal neutron signal return. Ancillary logging data, such as caliper logs for borehole diameter, information or porosity and density logs for formation definition, had not been obtained as a rule because of the limited capabilities of uranium logging service companies. Although probes which provide some of these logs are available, the uranium logging industry apparently does not generate sufficient demand for the service companies to provide them on a routine basis.

Borehole diameter information, obtained by caliper logs, is important because of the significant effect on the neutron signal produced by variations of water-filled borehole diameter.

The presence of hydrogen in the form of water in the formation can produce a significant change in the slowing down time of the $14 \mathrm{MeV}$ source neutrons because of the high scattering cross section of hydrogen. Changes in the slowing down time can have a significant effect on the epithermal neutron population as it is seen by the detector measurement time window. The net result will be epithermal count variations as a function of formation water which will distort the estimates of ore grade. For this reason, information in the form of porosity or density logs to define the quantity of formation water is essential.

The field data consist of both an analog and digital measurement of epithermal neutron returns. The digital data format represents average time resolved histories of the epithermal neutron population over nominal $15 \mathrm{~cm}$ depth increments while the analog data format is a continuous time record of the instantaneous epithermal neutron population gross count rate.

Digital data are considered the primary data format because of the expected advantage of interpreting formation effects as reflected in the epithermal neutron mean lifetime variations. It was believed that the neutron count for each digital datum point could be corrected for formation effects by use of the neutron mean lifetime alone or in conjunction with other formation information. So far, attempts to empirically define formation effects, as related to neutron mean lifetime only, have not proved successful.

The analog neutron record, which is the instantaneous gross count rate of the epithermal neutron population, was intended initially as a real time mineralization survey monitor to define zones for logging. However, the similarities between the PFN analog signal and the natural gamma signal provides an interesting look at the effect of disequilibrium. A comparison of the two recoras (logs) reflects the effect of disequilibrium since the neutron signal results from uranium within the sample volume while the gamma signal results from uranium daughters within the sample volume.

The sample volume of the neutron signal is defined, consistent with the practice for natural gamma signal, to be that volume from which originate approximately 99 percent of the neutrons captured by the detector. Although the sample volume for the PFN log will change more as a function of formation bulk density, porosity, and water saturation, theoretical estimates indicate it extends approximately $30 \mathrm{~cm}$ into the formation for typical formation parameters $\left(2.28 \mathrm{Mg} / \mathrm{m}^{3}\right.$ bulk density, 25 percent porosity, and 90 percent water saturation). The sample volume for natural gamma radiation in a similar formation is comparable.

The signal analogy also permits a similar data evaluation method for the two. The basic analytical tool for natural gamma logs has been the expression

$$
\overrightarrow{\mathrm{GT}}=\mathrm{KA}
$$


which relates the product of an average grade $(\bar{G})$ and a zone thickness (T) to the area (A) under the natural gamma count rate curve by a proportionality constant called the "K" factor. The grade thickness product $\bar{G}$ T is useful for estimating the economic value of ore zones.

However, for the purpose of comparative evaluation of the analog neutron and gamma logs, the grade thickness equation must be modified so the respective logs can be scaled and plotted on a common axis. If the mineralized zone is homogeneous and thick compared to the sample volume, the area of the count rate curve can be expressed as the product of a signal count rate (M) and a thickness (T) which permits the elimination of the thickness factor. The average ore grade of the homogeneous zone is then proportional to the signal count rate (M), for either neutron or gamma.

$$
\bar{\kappa}=\mathrm{KM}
$$

However, in nature ore zones generally are not homogeneous, and they will not always be thick compared to the sample volume. In the absence of these conditions, It the averagc ure graie $(\bar{G})$ is redefined as the instanlaneous average ore grade ( $\bar{g}$ ) within the sample volume, an evaluation of the analog neutron and gamma logs can be made for the purpose of comparing their response in conditions of disequilibrium. The ore grade, count rate expression

$$
\overline{\mathrm{g}}=\mathrm{KM}
$$

provides a convenient scale on which this analog comparison can be made and is not intended to imply precise grade estimates at any particular depth.

This equation expresses the ore grade $\bar{g}$ as a function of signal count rate (M) which is the true signal count rate. This definition is necessary because the true count rate (M) is not the quantity that is recorded on the strip chart record. The true count rate is modified by several factors, some of which can be corrected. The true signal count rate is altered hy the effects of borehole dlameter and fluid. The significance of this effect is rlearly shown in Figure 12. The data shown in Figure 12 wcre obtained with the hardware used during the feasibility study, which had a significantly diffcrent geometry than the prototype probe. The borehole water measurement was repeated using the prototype probe. In conjunction with computer neutron transport calculations, these data were used to generate a new factor for water-filled borehole attenuation.

$$
\mathrm{a}\left(\mathrm{d}, \mathrm{H}_{2} \mathrm{O}\right)=A e^{-B d}
$$

Equation (4) represents the attenuation factor for neutrons in a water-11lled luieliole where $A=2.20,1 / B=9.1 \% \mathrm{~cm}$ and $Q$ is the bultlule diameter (riguro 18).

The signal attenuation for both neutrons and gammas is minor for the case of a dry borehole. It is also a rare field condition; therefore, no further discussion will be devoted to it.

The gamma signal in a water-filled borehole is affected only slightly for a nominal $11 \mathrm{~cm}$ borehole, but its relative independence of borehole diameter is important. Changes in borehole diameter due to caving, washout, or other causes have only a minor effect on signal attenuation. This is the reason routine gamma log analysis does not require caliper log information. 
As seen in Figure 18, this is clearly not the case for the neutron signal. Borehole water severely attenuates the epithermal neutron population; more importantly the attenuation changes considerably with only a slight change in borehole diameter. The absence of caliper information to correct for small diameter changes introduces considerable uncertainty about the correction associated with borehole water. Unfortunately, the absence of caliper information during this period of field evaluation forces the use of a nominal constant diameter obtained from the drill bit size. Introducing the borehole correction modifies the instantaneous grade equation to

$$
\bar{g}=K M_{D}\left[\frac{1}{a\left(d, H_{2} O\right)}\right]
$$

where the signal count rate $\left(M_{D}\right)$ is now the signal count rate at the detector. The count rate at the detector would be the count rate of record except for count rate dead time losses. Dead time losses can be significant for natural gamma signal in high grade ores, but the neutron signal count rate is typically much lower and as a rule will not require correction. The count rate dead time correction is standard for natural gamma logs and it will be stated without definition. The correction for count rate dead time losses permits the statement of instantaneous ore grade in terms of the record count rate, for either neutron or gamma logs,

$$
\bar{y}=K\left[\frac{M_{R}}{I-M_{R} T}\right]\left[\frac{1}{a\left(d, H_{2} O\right)}\right]
$$

where $M_{R}$ is the count rate recorded on the analog record, and $T$ is the dead time.

Thus far, corrections that can affect both gamma and neutron logs have been included in : the ore grade expression. At this point, a correction unique to the neutron log is included."

If the source neutron generator output varies with time, the epithermal neutron population, and hence the neutron signal count rate, will vary proportionally if all. other conditions remain constant. The neutron generator reservoir control provides good short term stability, but in the long term (tens of minutes) the generator output is constantly decreasing. The neutron signal level must be compensated for the neutron generator output reduction. That correction is made by observing the neutron output rate change that occurs with time for a fixod neutron output.

The source neutron total. output for a digital datum point is $10^{9}$ neutrons. The time required for the neutron source to generate $10^{9}$ neutrons at logging time is compared to the time required at calibration and the ratio of the two time intervals corrects for the loss of the neutron source output rate. The grade expression with this correction is

$$
\bar{g}=K\left(\frac{M_{R}}{1-M_{R} T}\right)\left(\frac{1}{a\left(d, H_{2} O\right)}\right)\left(\frac{T_{L}}{T_{C}}\right)
$$

where $\mathrm{T}_{\mathrm{L}}$ is the time interval during the logging run and $\mathrm{T}_{C}$ is the time interval at calibration. This correction can be thought of as a " $K$ " factor correction since the $K$ factor is really a function of the neutron source output rate. For a gamma log, this ratio will always be one.

This grade equation represents all of the corrections that can be made to the data 
acquired during the field evaluation period ending in June 1976. The neutron and gamma logging records must be scaled to account for the corrections included in Eq. (7). A calculator with digitizer, magnetic storage, and plotting peripherals (13) was used to establish a permanent file on magnetic tape and scale and replot the records.

Although the comparison of the two corrected analog records was justified on the basis of relative rather than absolute response to borehole mineralization, an independent ore grade estimate derived from core sample analysis will be included in histogram form in order to provide a reference for the mineralization in the borehole. A comparison between the neutron or gamma logs and core sample ore grade must be considered carefully because of the relative difference in their sample volumes. Core samples are right circular cylinders 15 to $30 \mathrm{~cm}$ in length and $11-12 \mathrm{~cm}$ in diameter, considerably smaller than sample volumes for the neutron and gamma logs. Because of the smaller sample volume of core samples, narrow ore zone definition will be more precise, while the larger sample volumes of the neutron and gamma logs will tend to average narrow zone mineralization with adjacent barren material, producing a lower ore grade indication. Narrow zone mineralization can pluduce vinjiderable disagreement between the logs and the core, sample histogram, while thick zones may agree very closely.

A correction for the narrow ore zone effects has been deferred until better analytical definition of the neutron signal sample volume can provide more complete information suitable for the derivation of a correction factor.

Figures 19 through 33 include a complete record of all borehole logs made during this period for which core analyses are currently available. All of these logs, neutron and gamma, were scaled using Eq. 7 .

\section{Discussion and Conclusions}

In most comparisons of the analog neutron and gamma logs, the conclusions are currently more qualitative than quantıtative because uf Lhe absence of oufficiont fnrmatinn and diameter information for precise correction of the raw data. The calibration test pit (Fig. 7), for simplicity and consistency, was constructed with low density $\left(\because 1.5 \mathrm{Mg} / \mathrm{m}^{3}\right) \mathrm{and} 10 \mathrm{w}$ formation moisture (< 1 percent $\mathrm{H}_{2} \mathrm{O}$ by weight) content, which is a relatively uncommun condition for uranium exploration borehole surroundings. The effect of the difference between the test pit "formation" and typical exploration borehole formations lcads to significant error. In conjunction with the errors related to unknown borehule diameter variations, substantial grade error can occur which cannot be identified as relating to one or the other effect. The most significant depth digit has been deleted from the comparison plots for proprietary reasons.

Figures 19 and 20 were made at a site in Arizona at whlch the fuimalion moijture was extremely low and the boreholes were drilled dry. This formation, of all those encountered in the field, was most similar to the conditions of the calibration pit. Because the borehole was drilled dry and air-filled when logged, changes in the diameter are insignificant (Fig. 18). As a result of this lorlunate similarity of conditions, the neutron logs agree extremely well with core grade for the thick zones. The narrow zones do not agree because of the sample volume difference discussed earlier.

In both figures, the effect of disequilibrium, reflected in the difference between the neutron and gamma logs, is clearly evident. In both holes, uranium mineralization has been 
substantially leached from the wide, shallower zone and perhaps deposited in the thin lower zone which has yet to reach the equilibrium condition. These two figures probably best represent the potential of the PFN logging technique for not only detecting disequilibrium but primarily grading uranium ore accurately.

Figure 21 indicates a condition of near equilibrium, a condition not uncommon in the Grants Mineral Belt. Figure 22 in the same region, indicates a clear disequilibrium situation. Absolute ore grade estimates in both boreholes are limited because both were filled with drilling liquid and no caliper logs were run.

Figures 23 and 24 are logs of large diameter holes in preparation for leach field operation. Figure 23 is affected more by the narrow-zone effect but clearly shows disequilibrium. Figure 24 is a $\log$ in a borehole already cased for leaching, which creates considerable uncertainty as to the effective diameter because of the screen mesh in the leach zone.

Figures 25 and 26 are logs from an area that exhibited poor formation stability. Caving often occurred within a few minutes after drilling, forcing washout operations which made borehole diameter estimates impossible without caliper logs. The neutron log clearly responds to the uranium mineralization as defined by the core grade and shows small regions of disequilibrium, but the narrow-zone sample volume problem is evident in combination with borehole diameter uncertainties produced by washout. Figure 26 in particular shows a wide region (the upper zone) that cannot be explained by the narrow-zone effect and likely therefore to be due to substantial washout.

Figures 27 through 30 are exploration boreholes in the same region. Formation instability again caused uncertainty in diameter estimates and therefore grade estimate accuracy. Figures 28 and 30 show disequilibrium, in particular Figure 30 . Figures 31 through 33 are cased leach holes on the same site. The significant feature of these three logs is the considerable discrepancy between the core grade and the neutron logs. The fact that the leach holes were initially drilled large for casing and had been standing for several months complicates any diameter estimate for borehole fluid correction.

The salient features of these logs are first a clear definition of disequilibrium and second the consistency with which the neutron record follows the formation variation defined by the core samples. The fact that significant absolute ore grade discrepancies exist for the bulk of these records does not at this point detract from the PrN logging technique. Although no serious attempt can be made to estimate the absolute accuracy with which ore grade estimates can be made at this time, it is clear the PFN logging technique can offer significant advantages over natural gamma logs even at its current rudamentary stage of development. It is also clear that considerable improvement in absolute grade accuracy is possible in the near future with the aid of caliper logs, formation logs, and the necessary theoretical definition of the sample volume to produce an accurate narrow zone correction. 
NEUTRON POPULATIONS AS A FUNCTION OF TIME AND ENERGY.

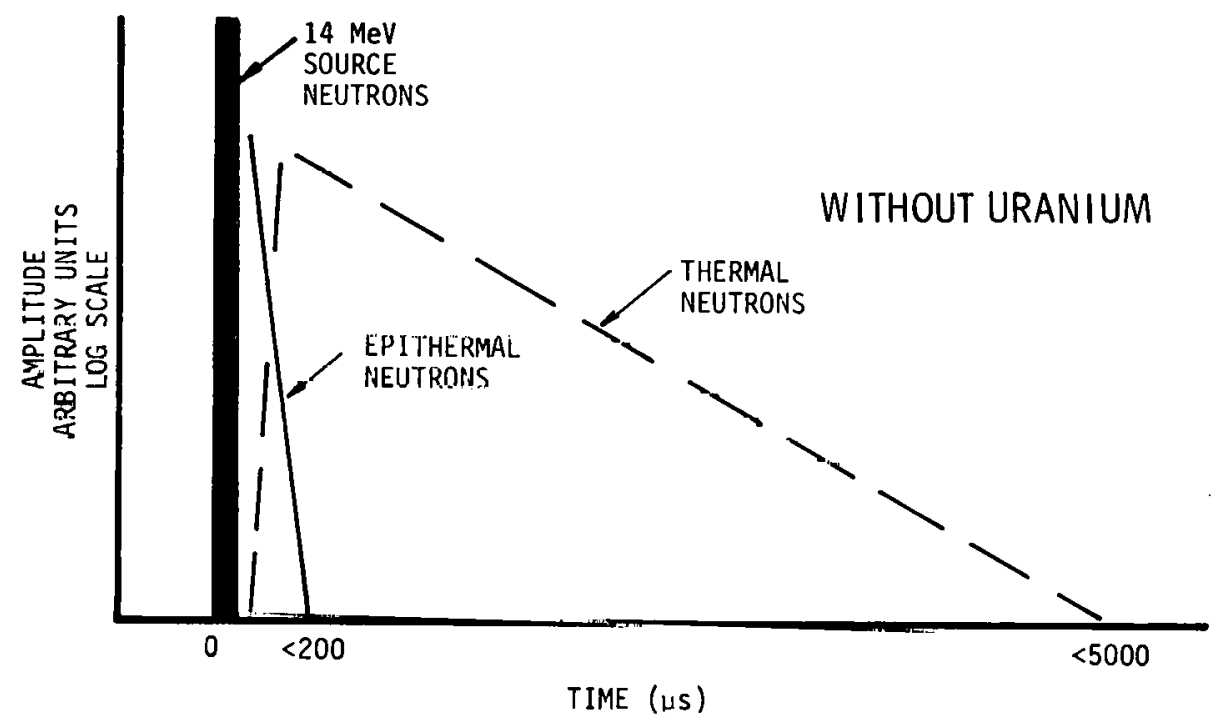

FIG 1 .

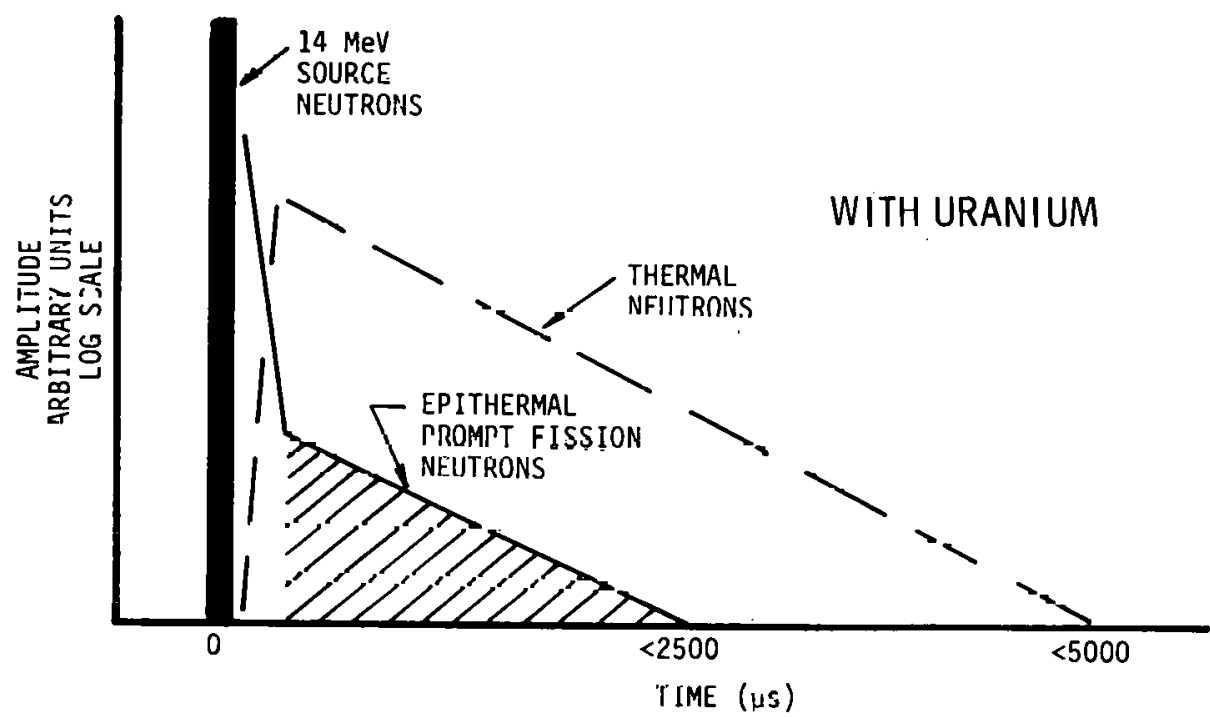

FIG 2. 


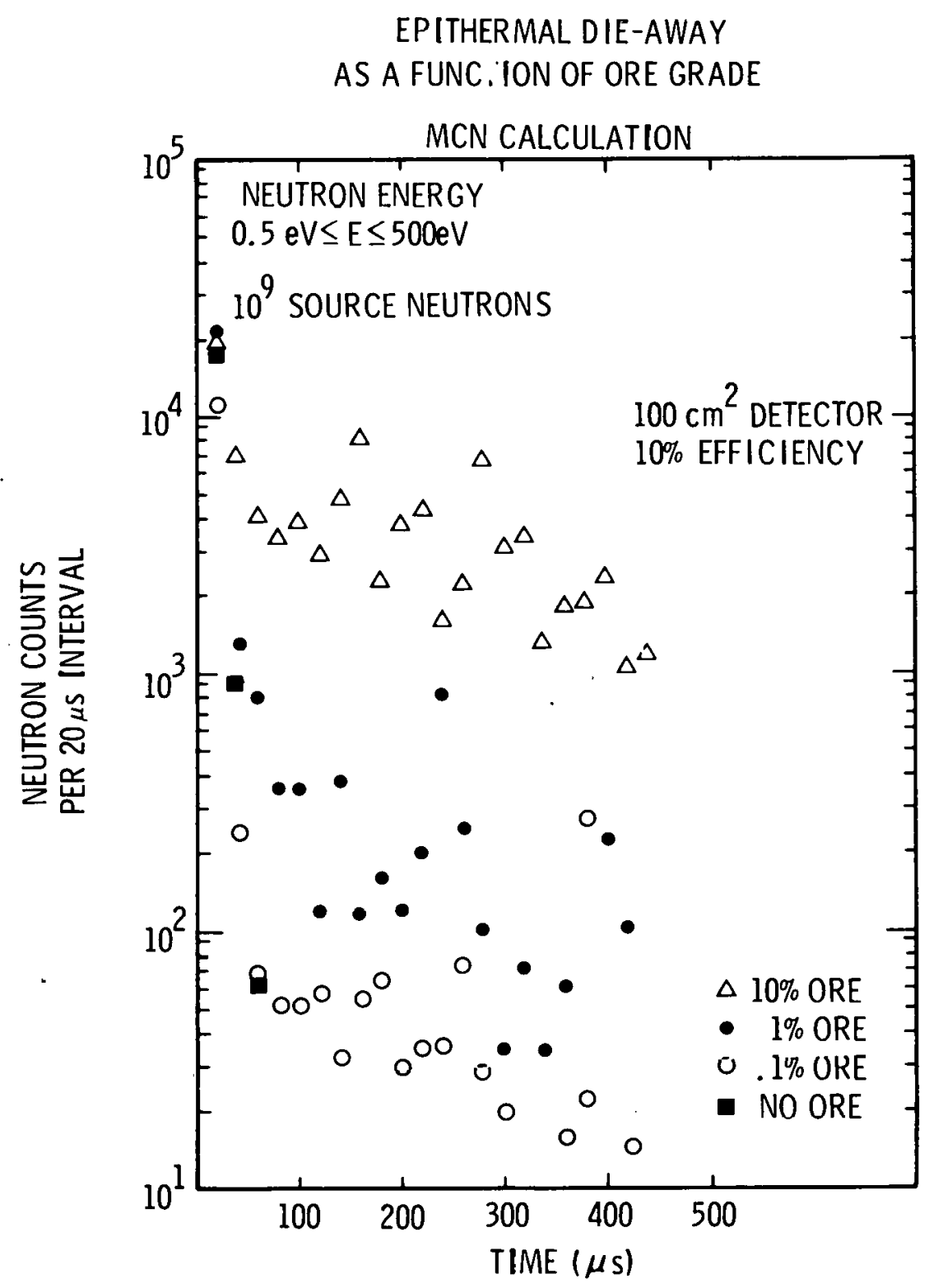

FIGURE 3. 


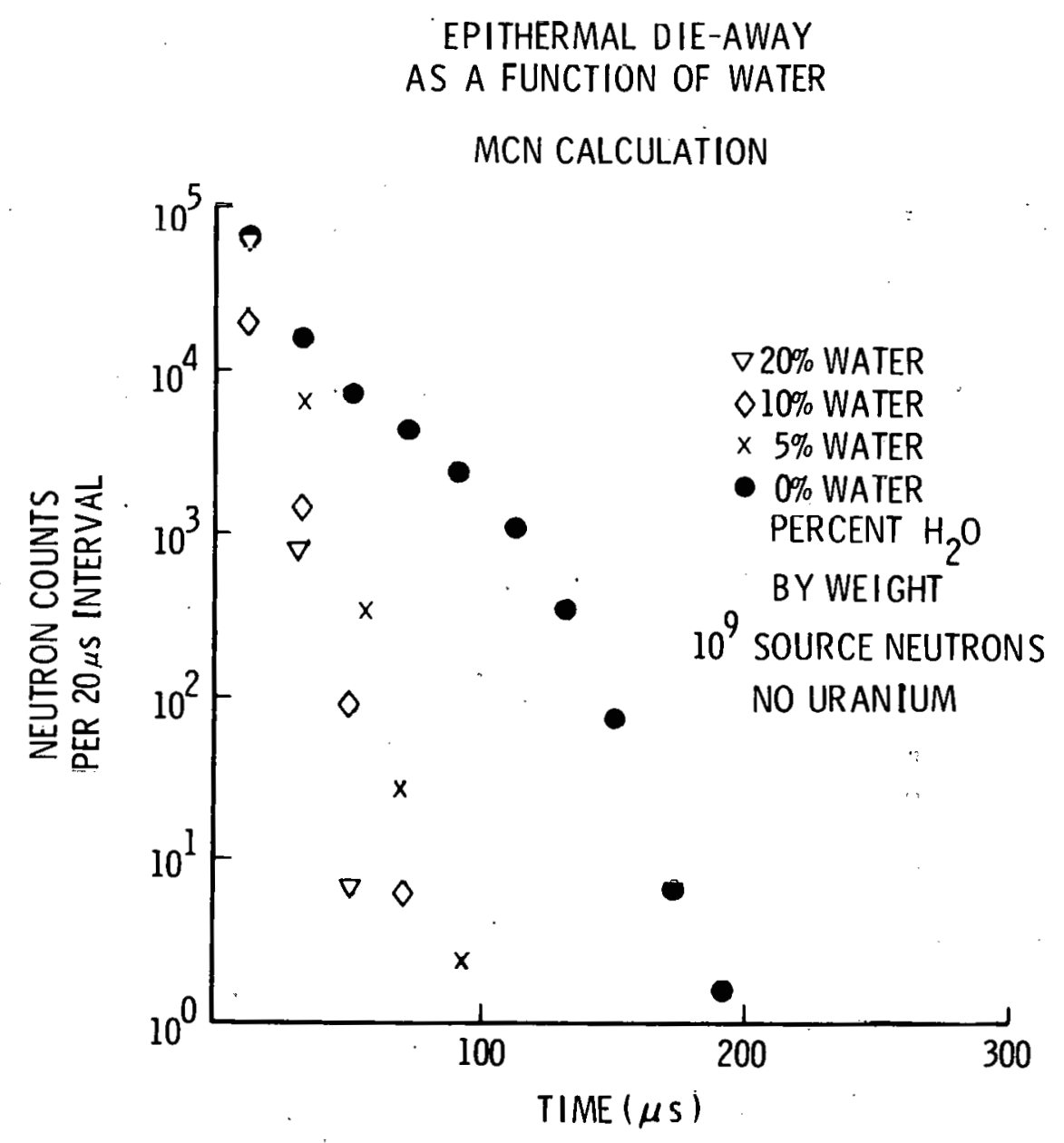

FIGURE 4. 
EFFECT OF NEUTRON ABSORBER̉S ON THERMAL NEUTRONS

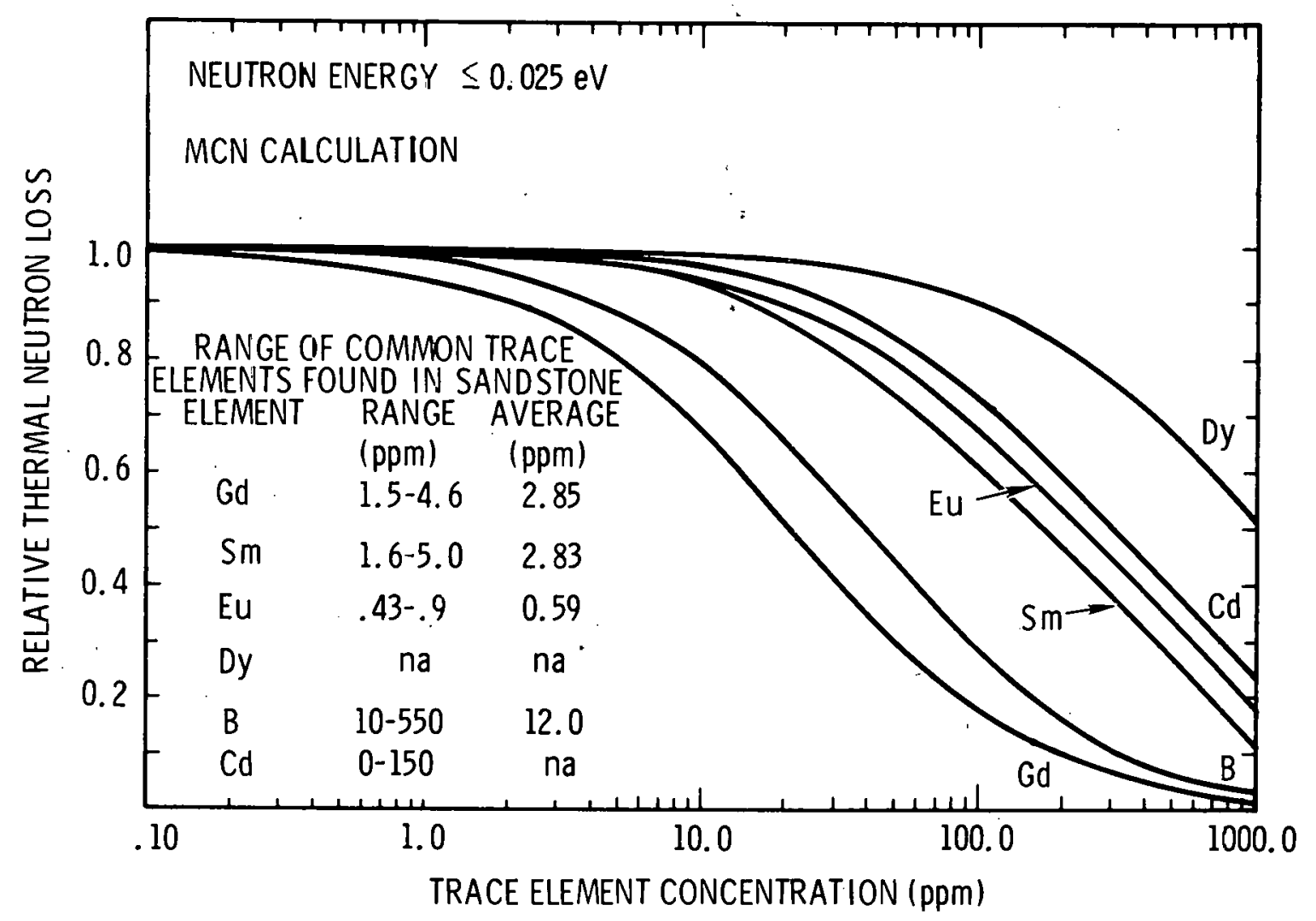

FIGURE 5. 


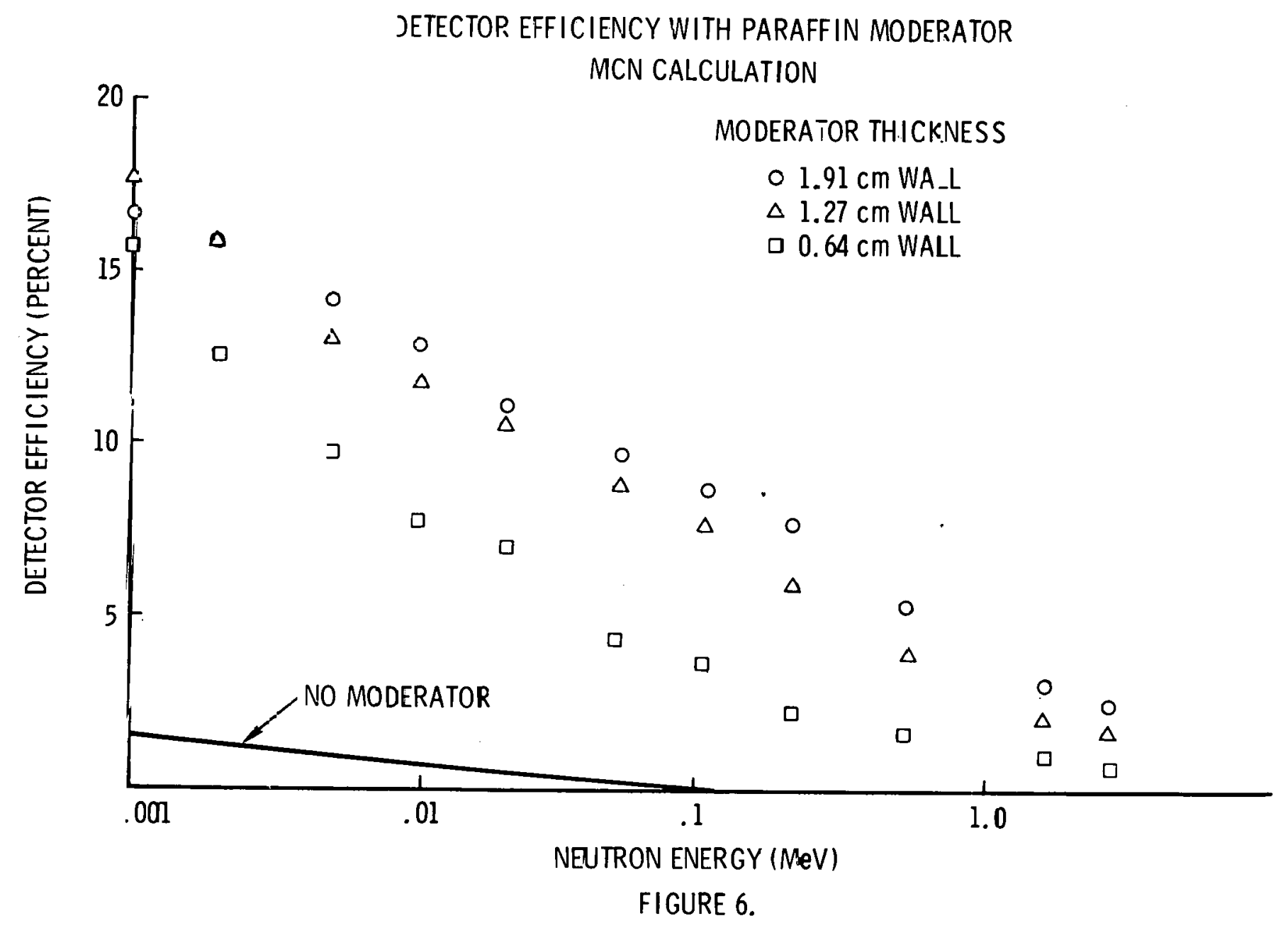


EXPER IMENTAL BOREHOLE CONFIGURATION

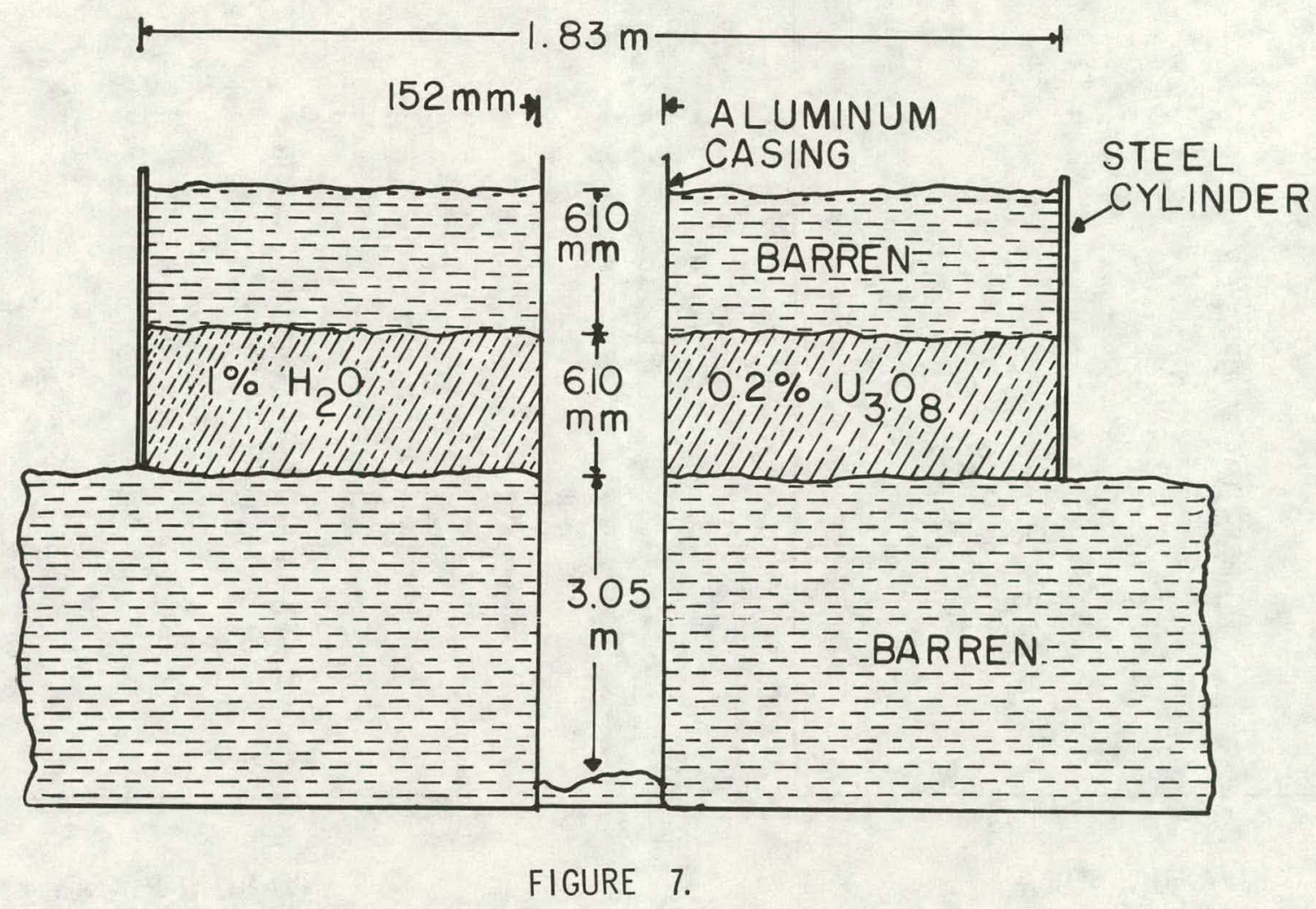


EXPER IMENTAL BOREHOLE TESTS

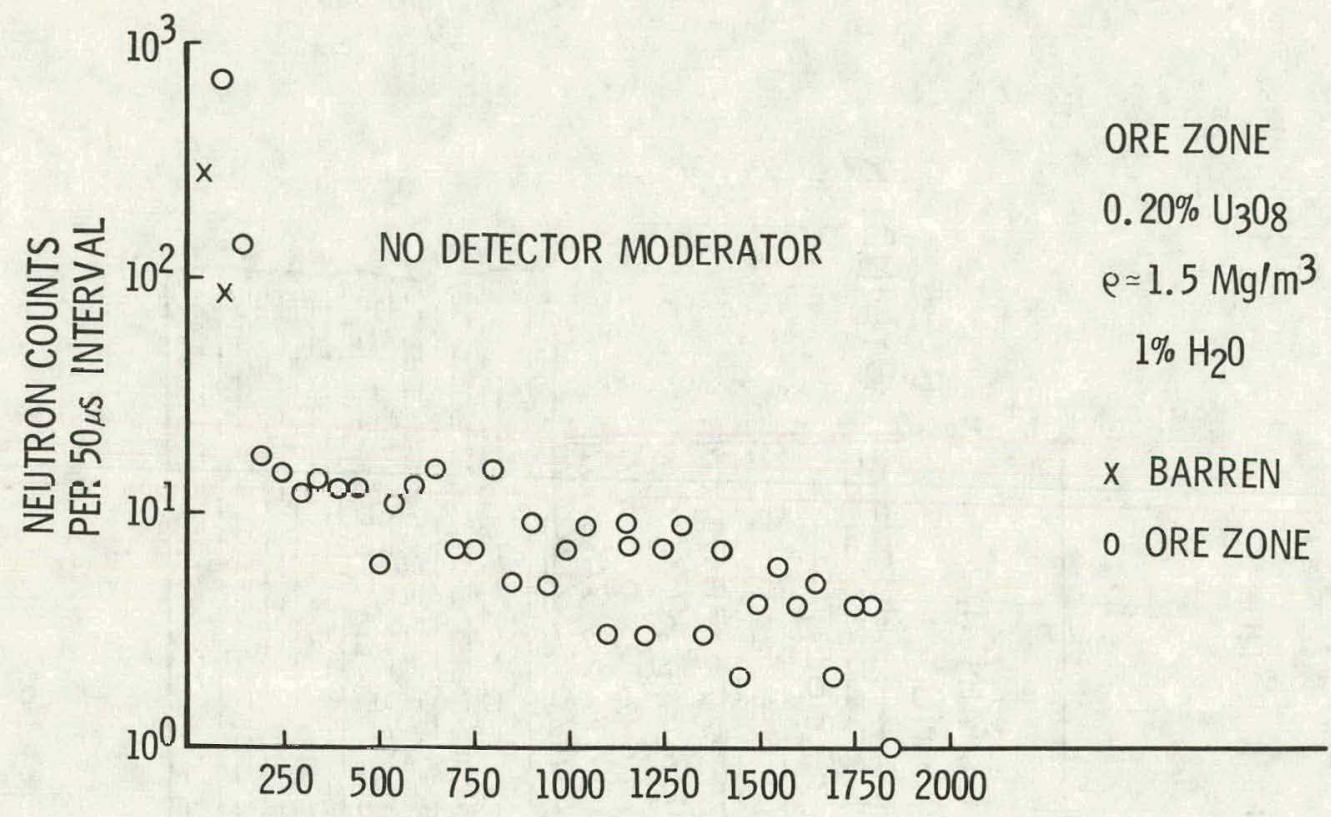

TIME ( $\mu$ s)

FIGURE 8

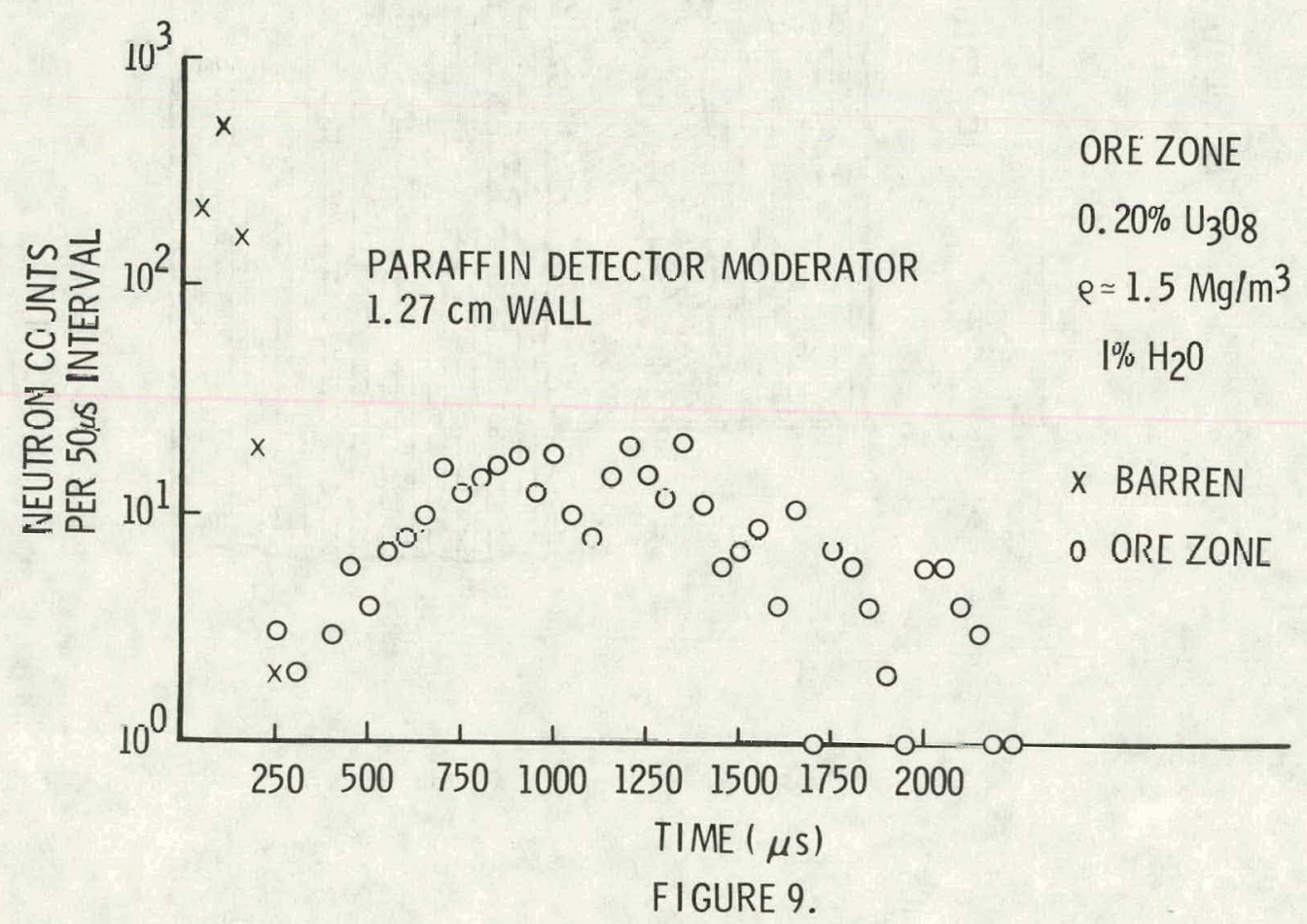




\section{DETECTOR OVERLOAD *}

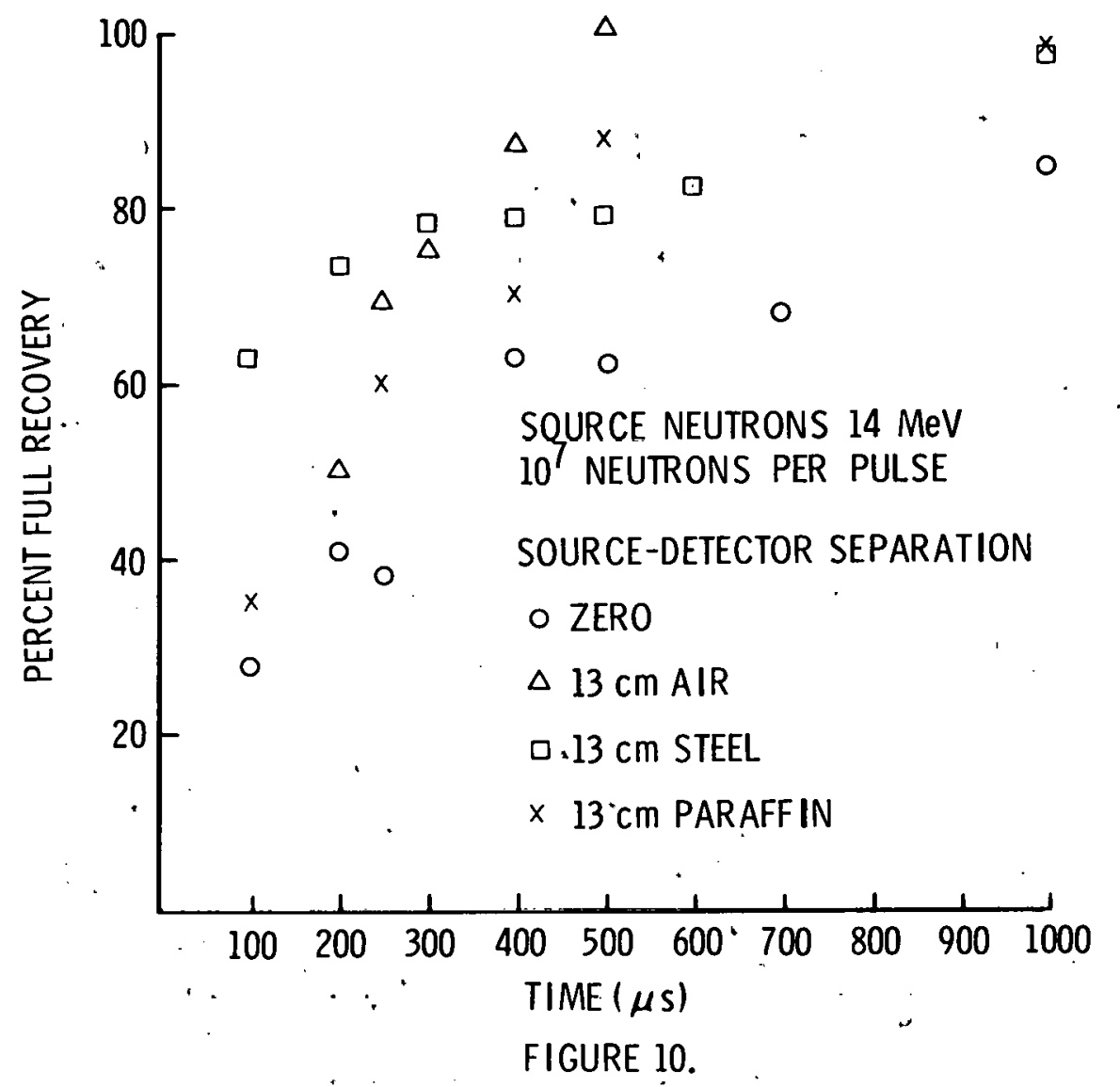




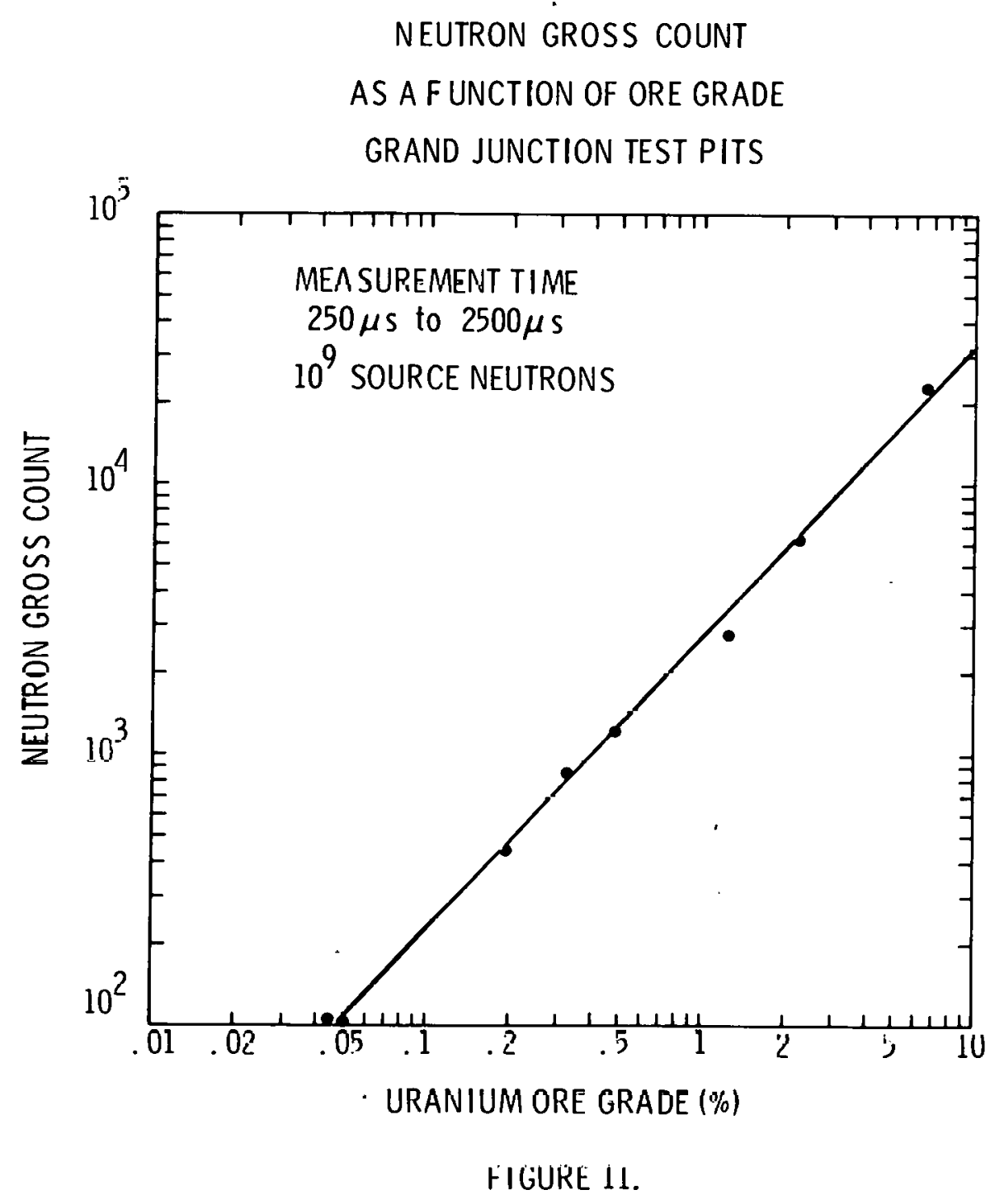




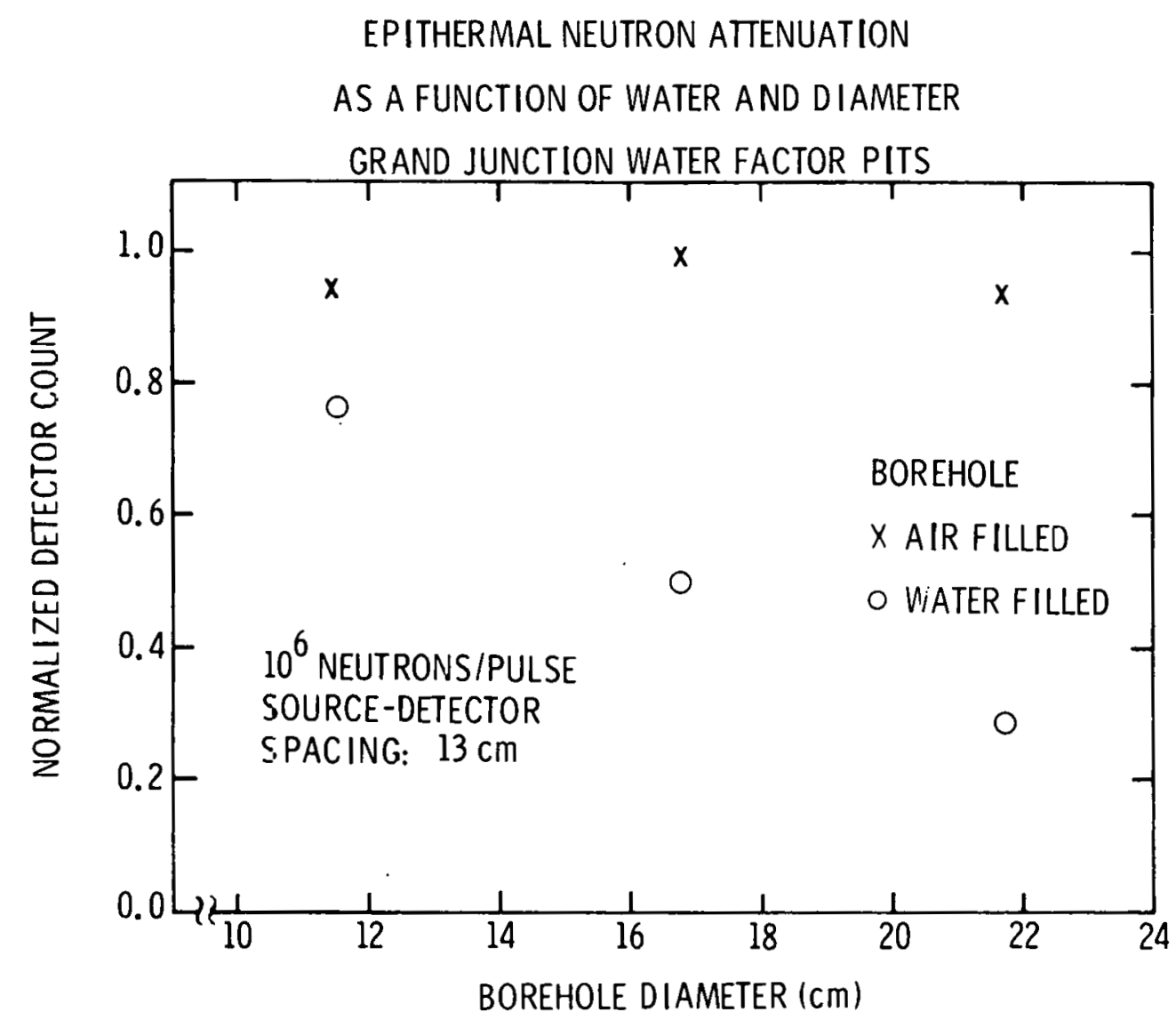

FIGURE 12. 


\section{DETECTOR OVERLOAD}
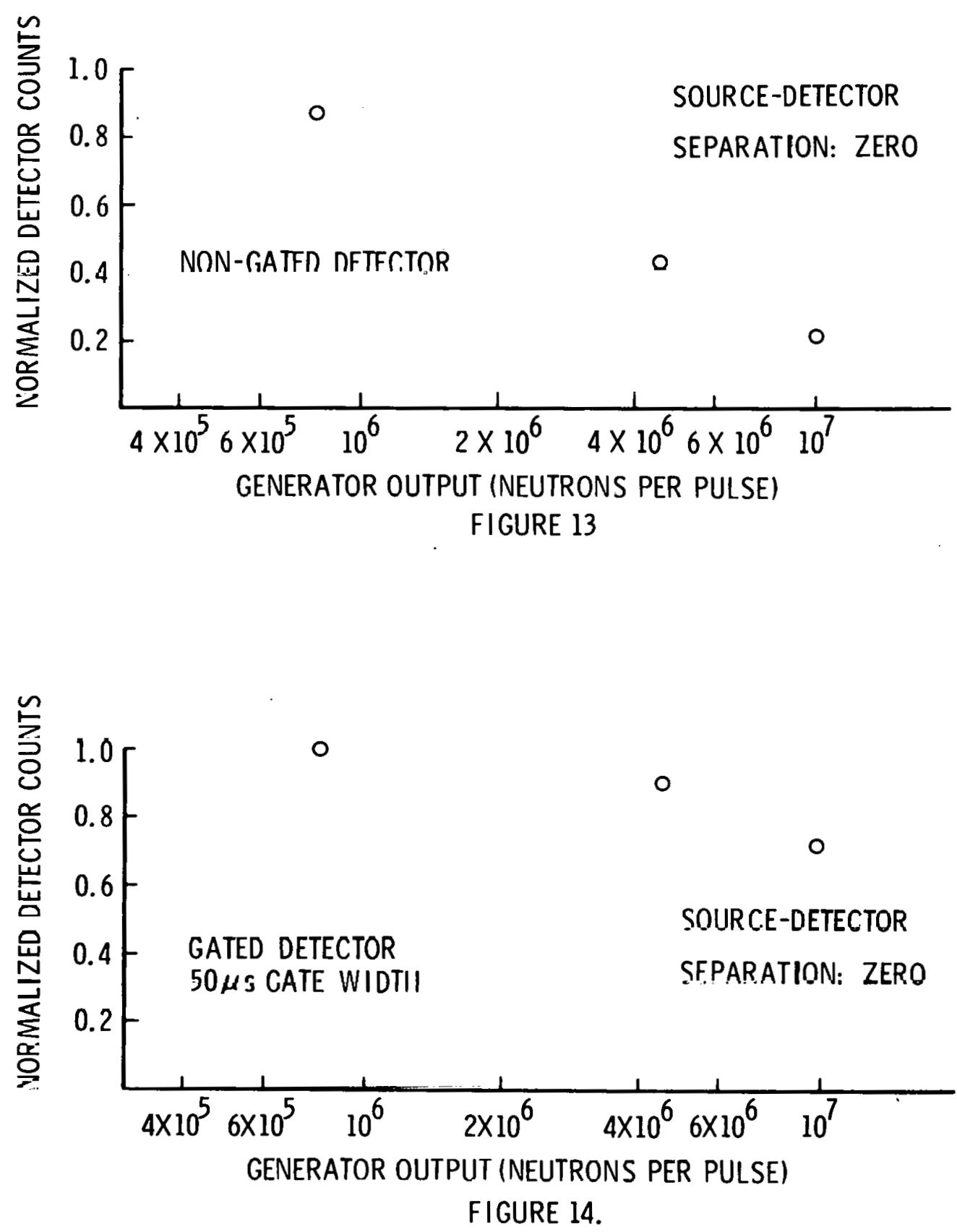


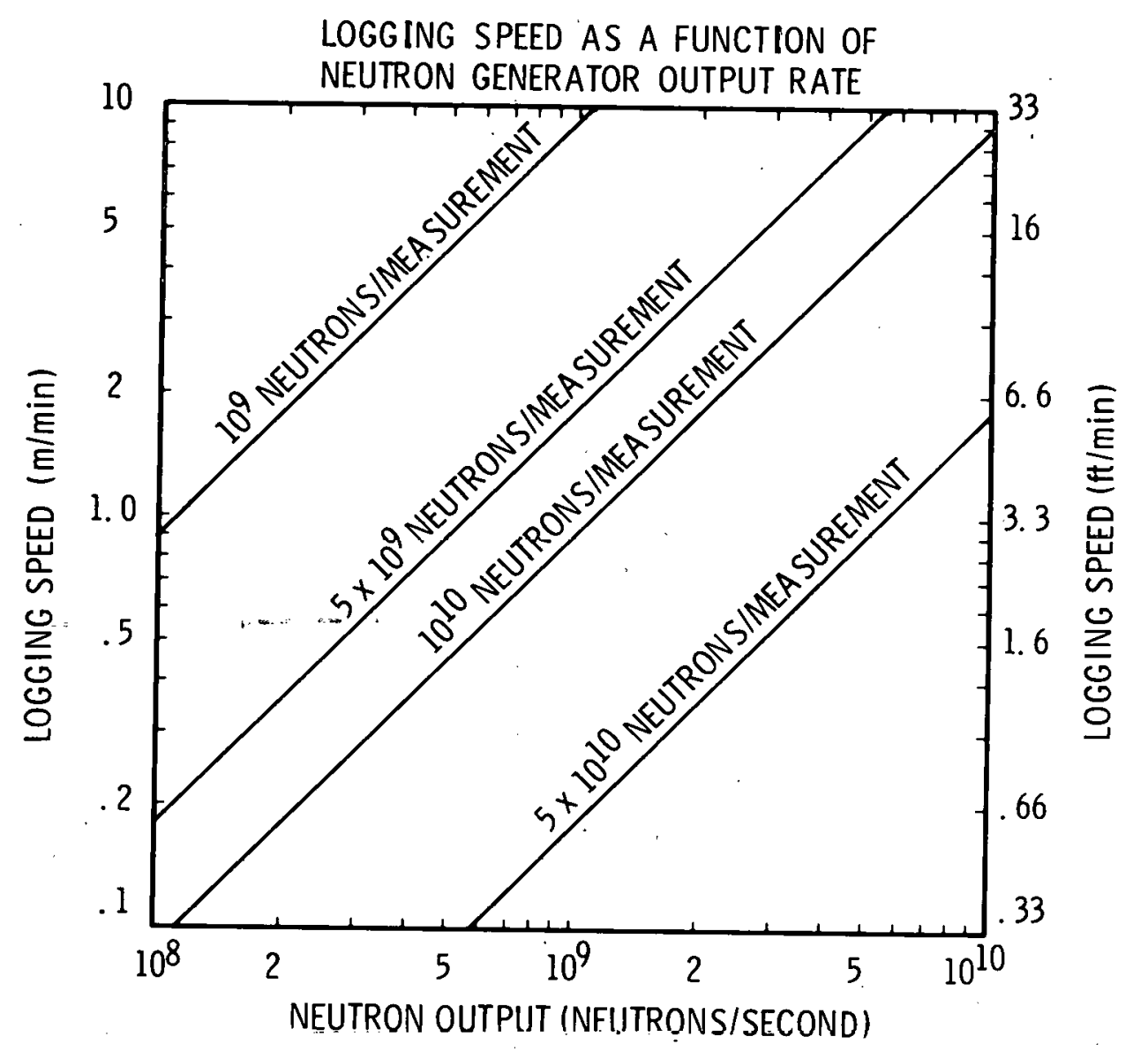

FIGURE 15 
IFN PROTOTYPE PROBE

\begin{tabular}{|c|c|c|c|c|c|}
\hline $\begin{array}{l}{ }^{3} \mathrm{He} / \mathrm{Cd} \\
\text { DETECTOR } \\
685 \mathrm{~m}: \mathrm{m}\end{array}$ & \begin{tabular}{|c|} 
TUBE \\
TRANSFORMER \\
A SSEMBLY \\
$255 \mathrm{~mm}$
\end{tabular} & $\begin{array}{l}\text { SCURCE } \\
\text { NEUTRON } \\
\text { MDNITOR } \\
305 \mathrm{~mm}\end{array}$ & $\begin{array}{l}\text { PULSE } \\
\text { FORMING } \\
\text { NETWORK } \\
610 \mathrm{~mm}\end{array}$ & $\begin{array}{l}\text { D.C. - D.C. } \\
\text { 2ONVERTER } \\
570 \mathrm{~mm}\end{array}$ & $\begin{array}{l}\text { RESERVOIR } \\
\text { CONTROL } \\
305 \mathrm{~mm}\end{array}$ \\
\hline
\end{tabular}

FIGURE 16. 
POWER

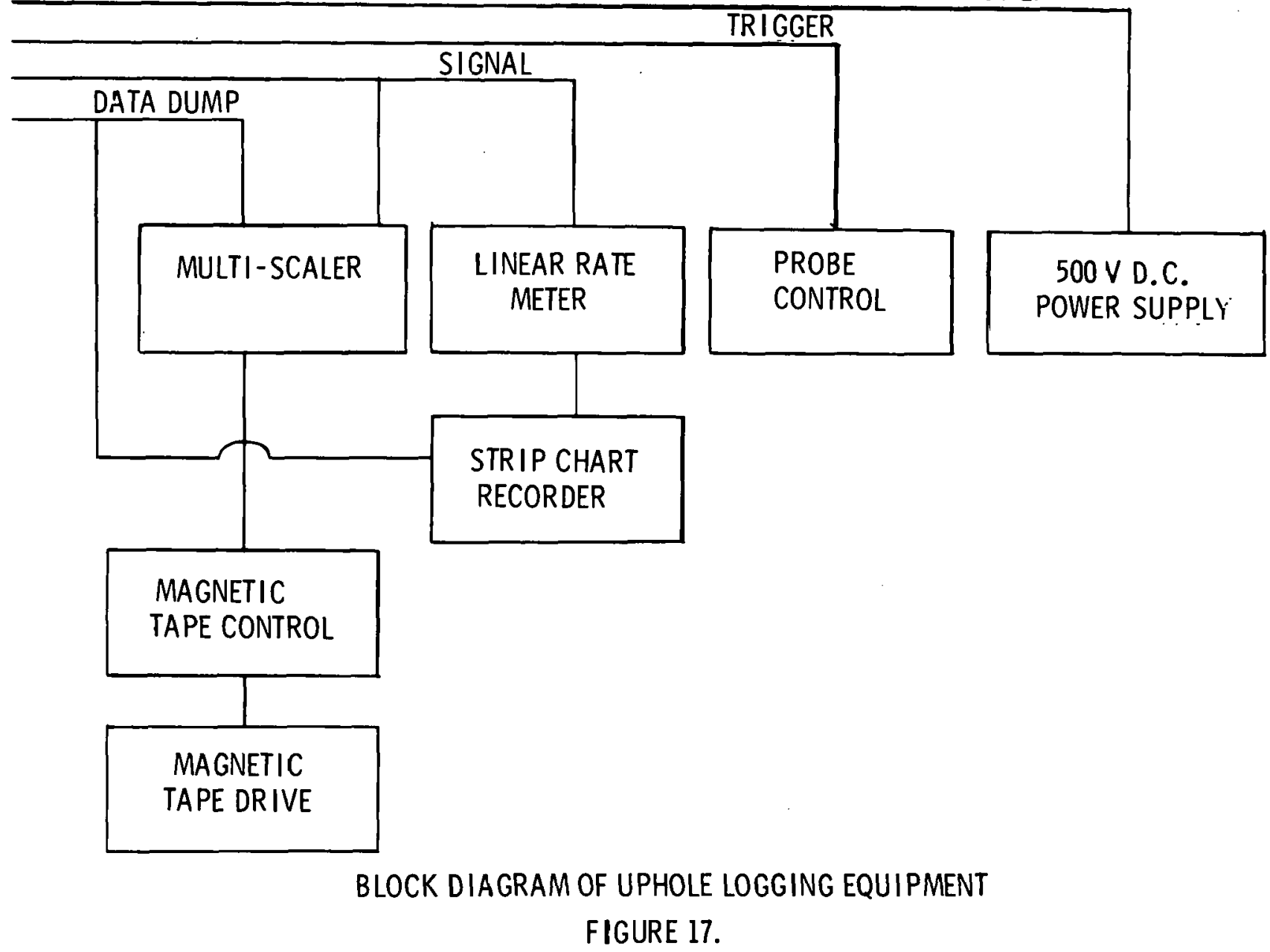

FIGURE 17. 


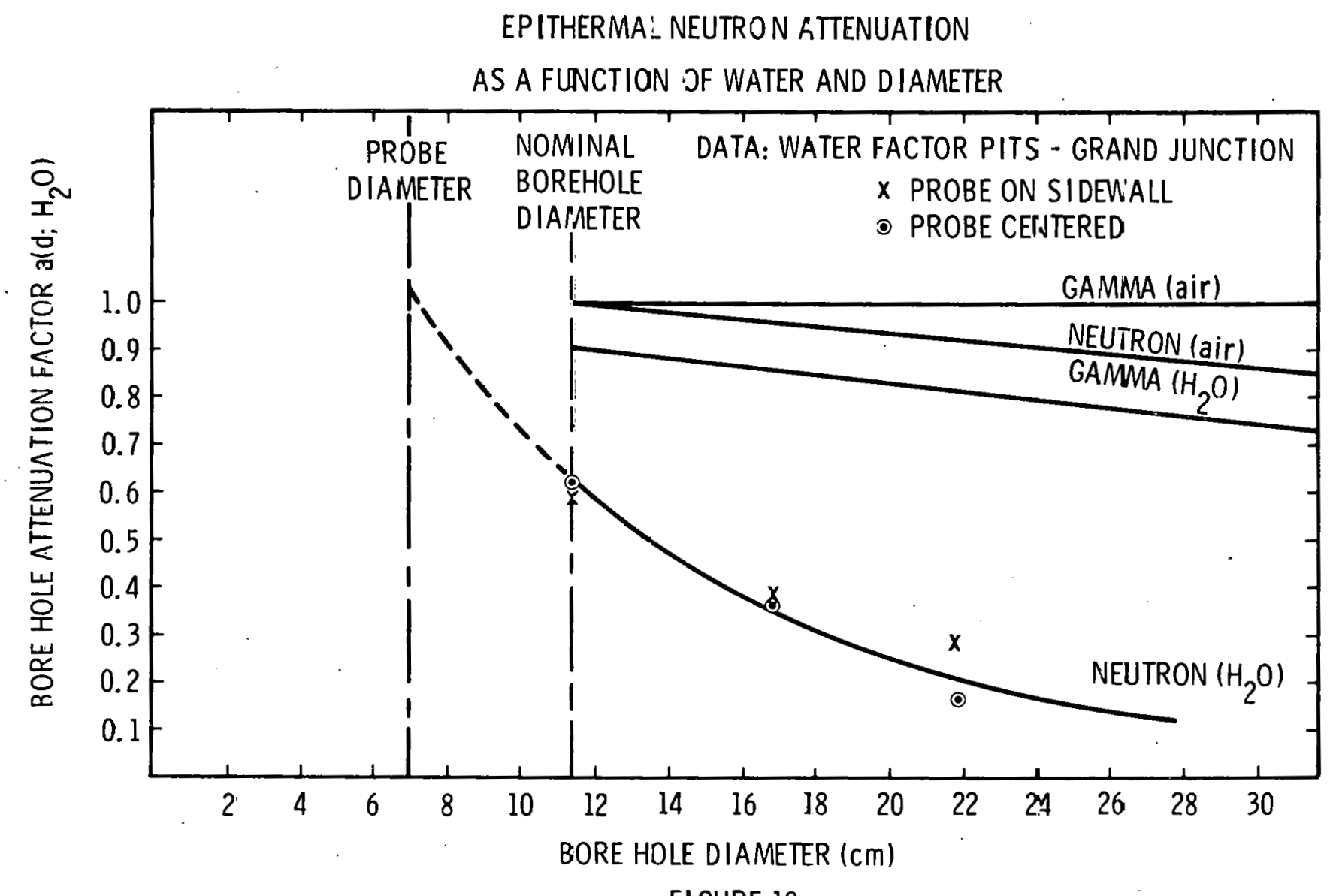

FIGURE 18. 


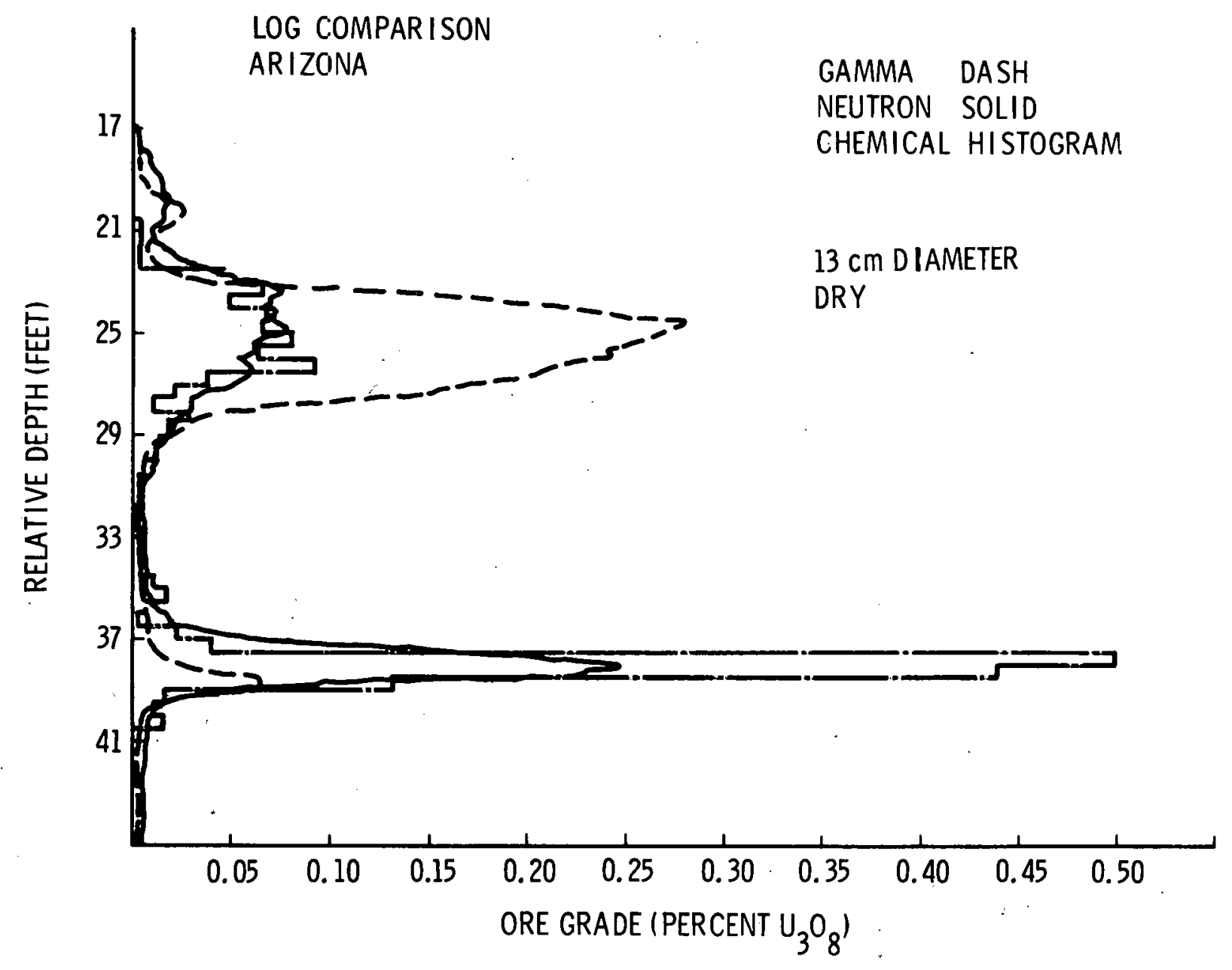

FIG: 19 


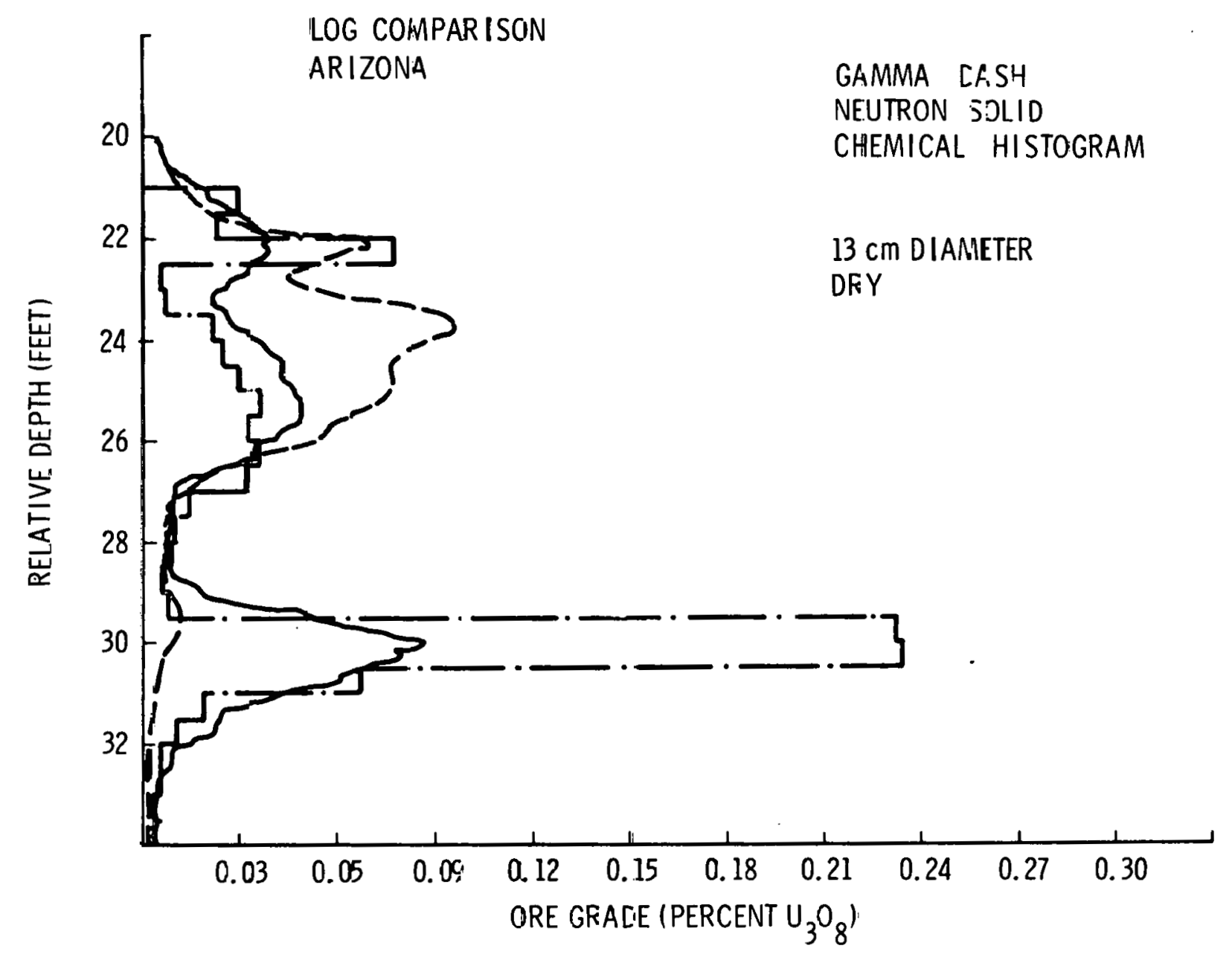

FIG 20 


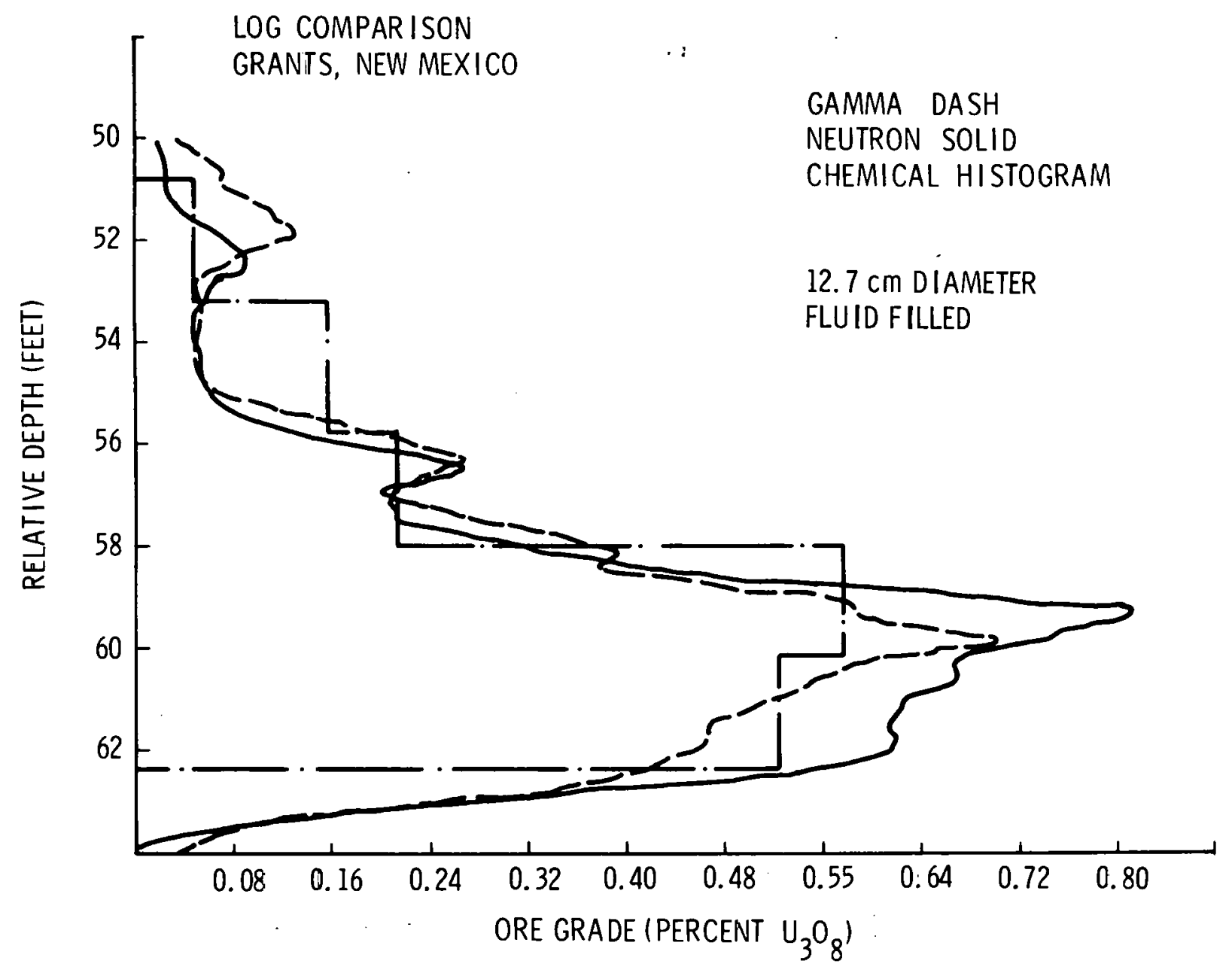

FIG. 21 


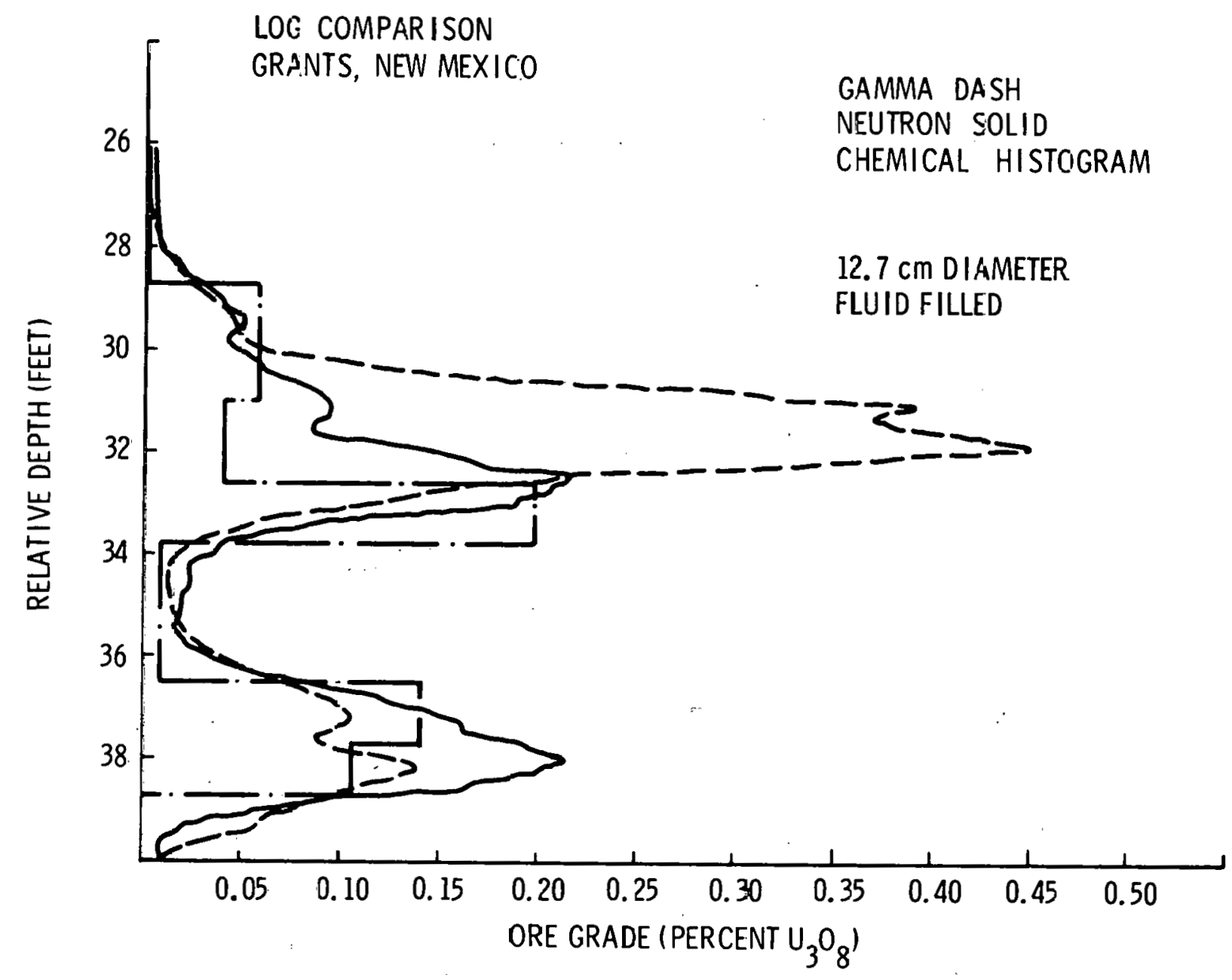

FIG. 22 


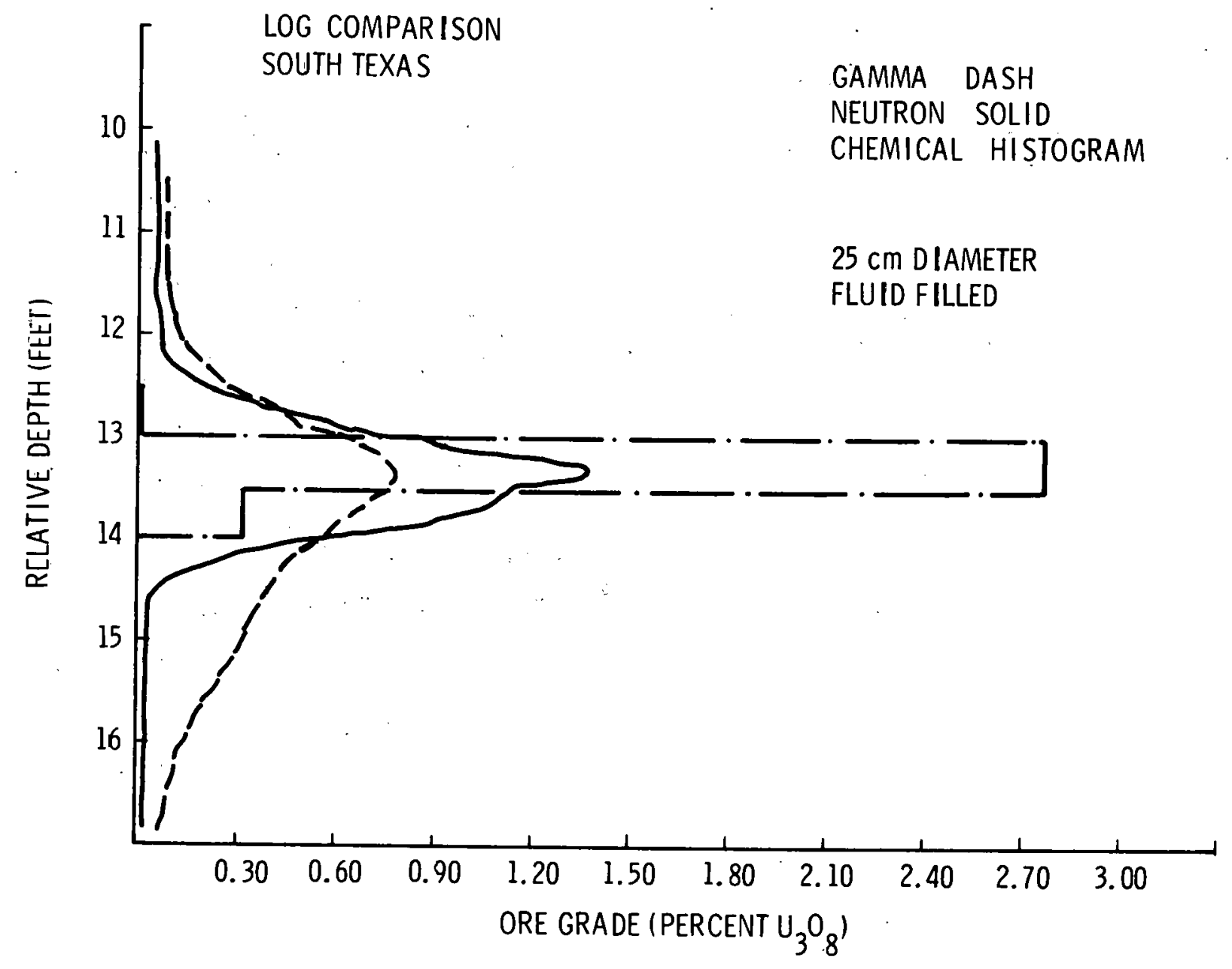

FIG. 23 


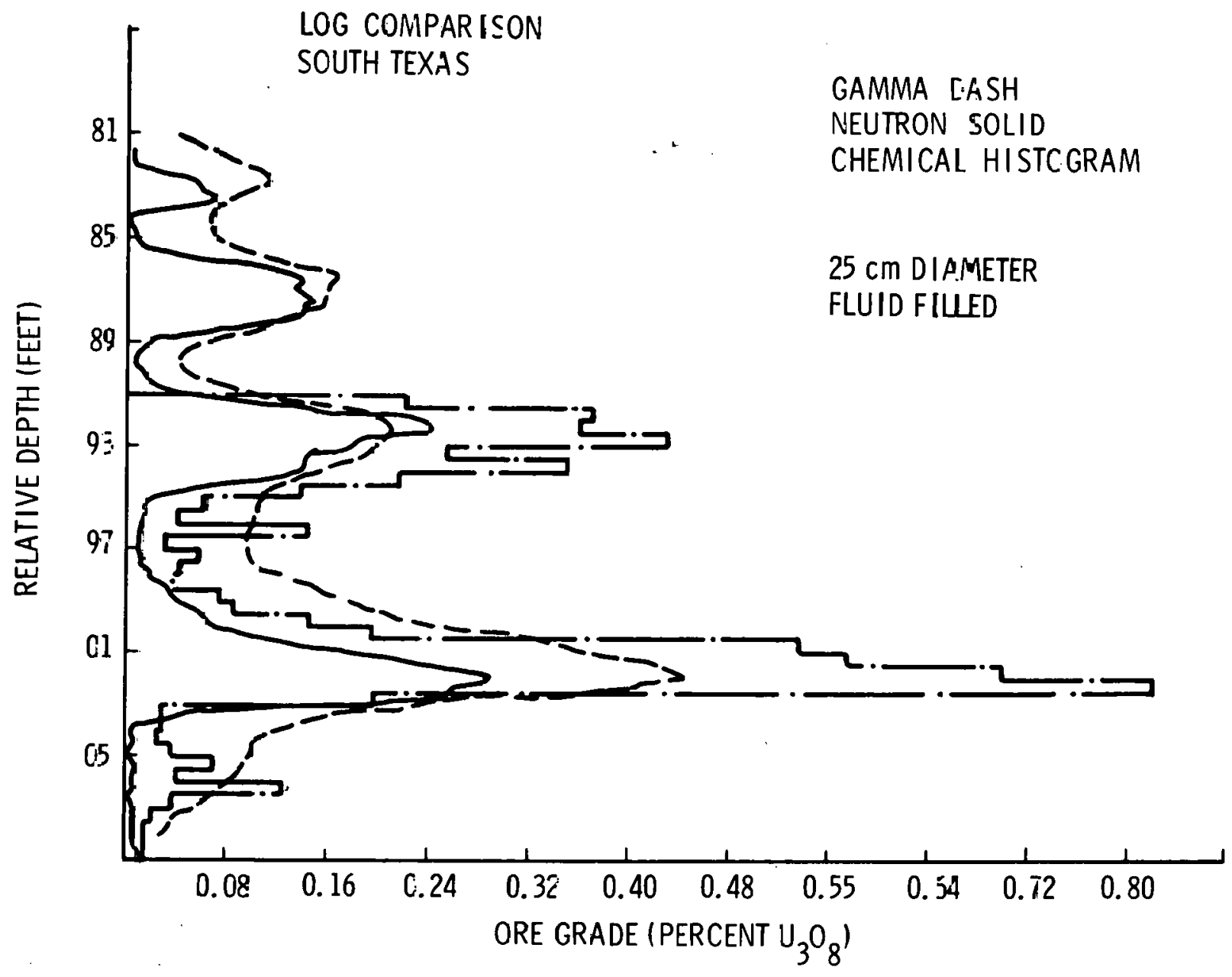

FIG. 24 


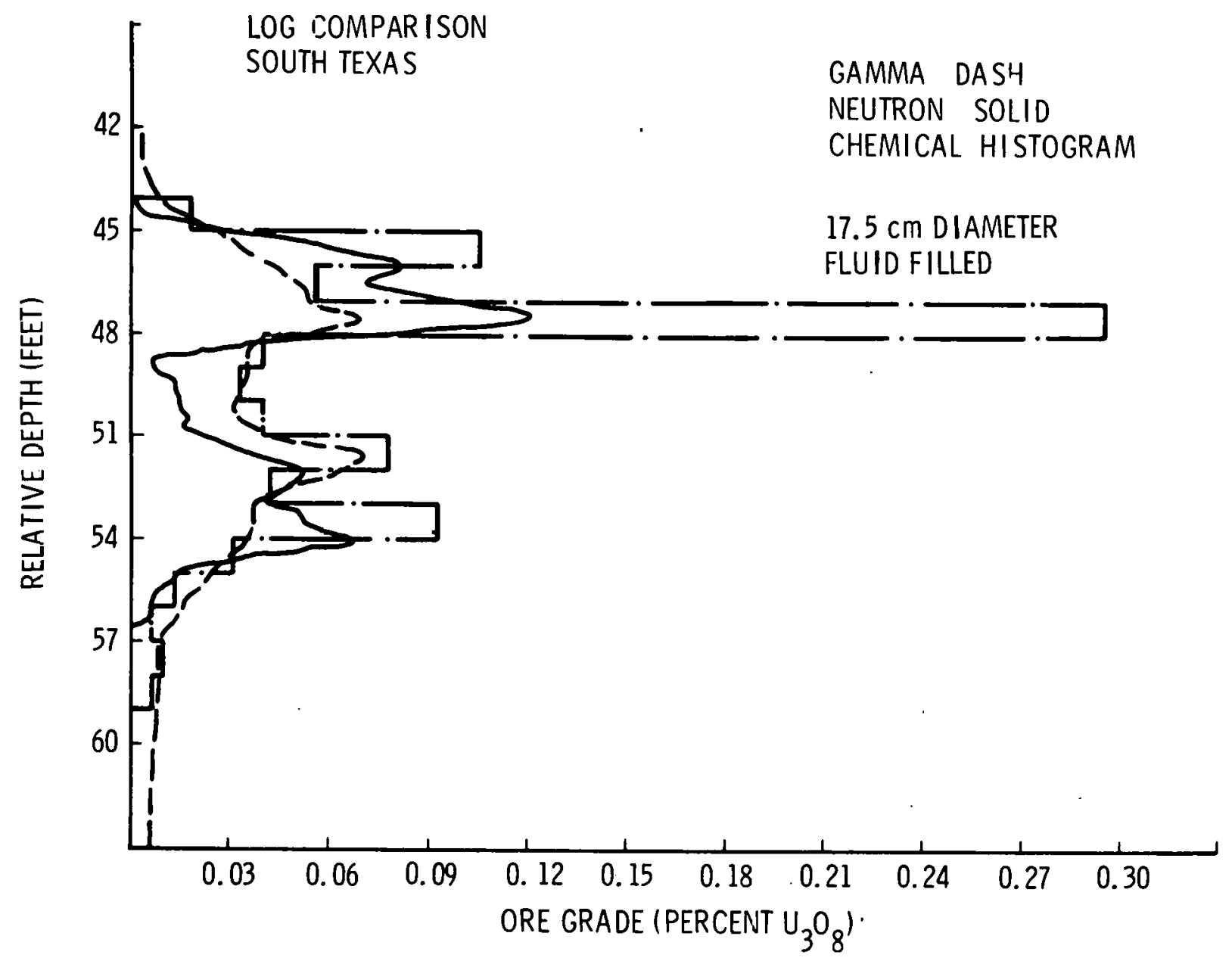

FIG. 25 


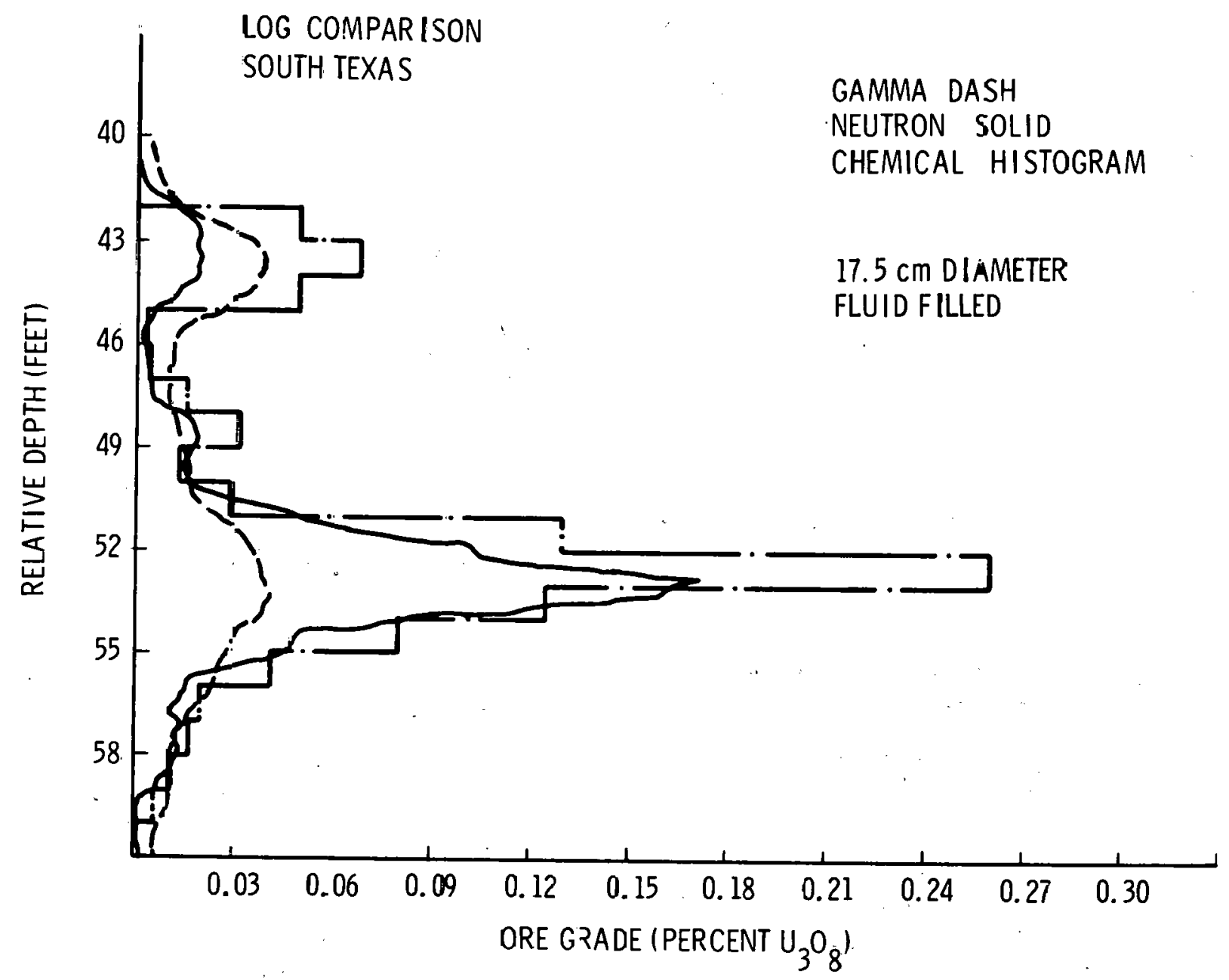

FIG. 26 


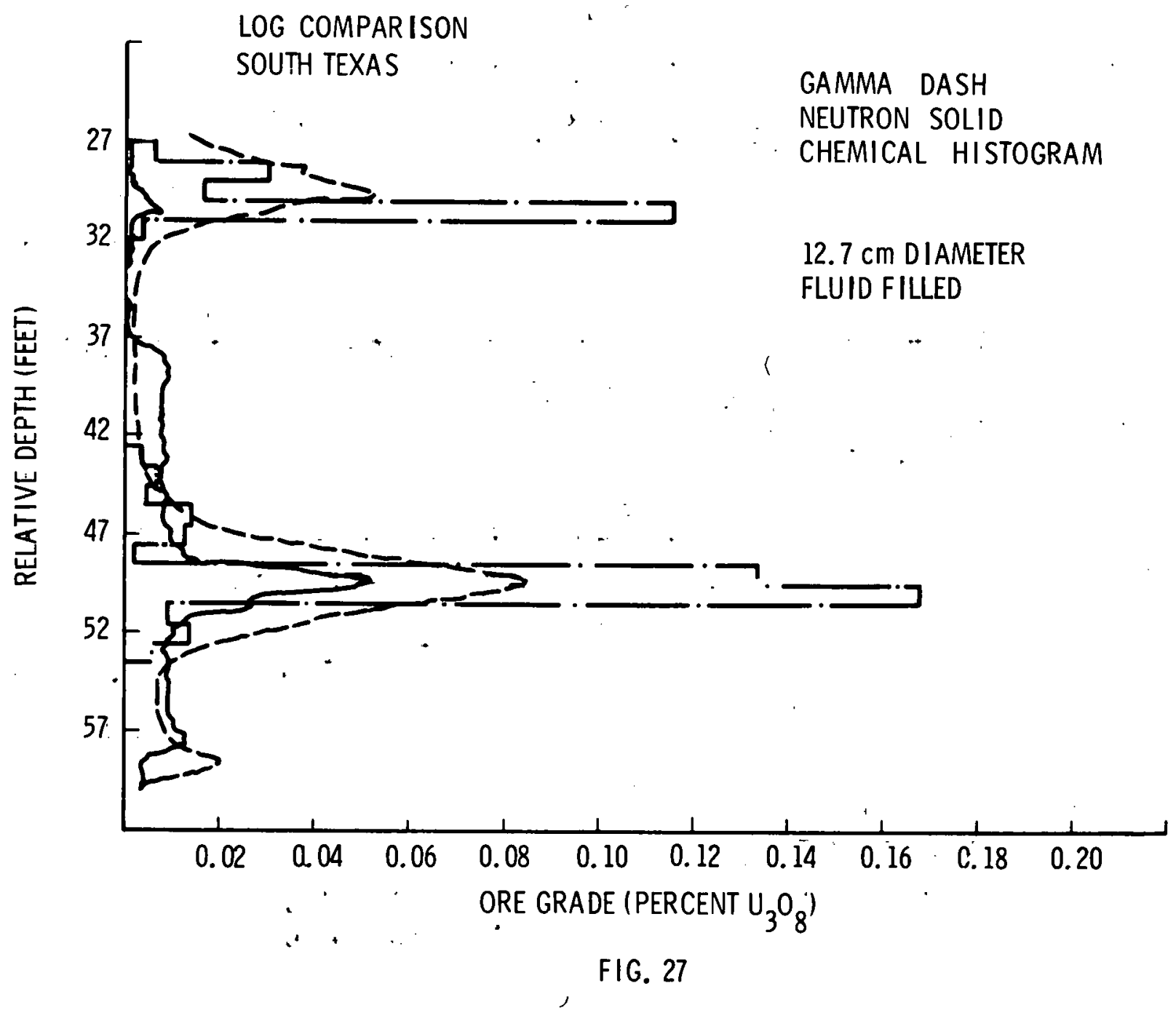




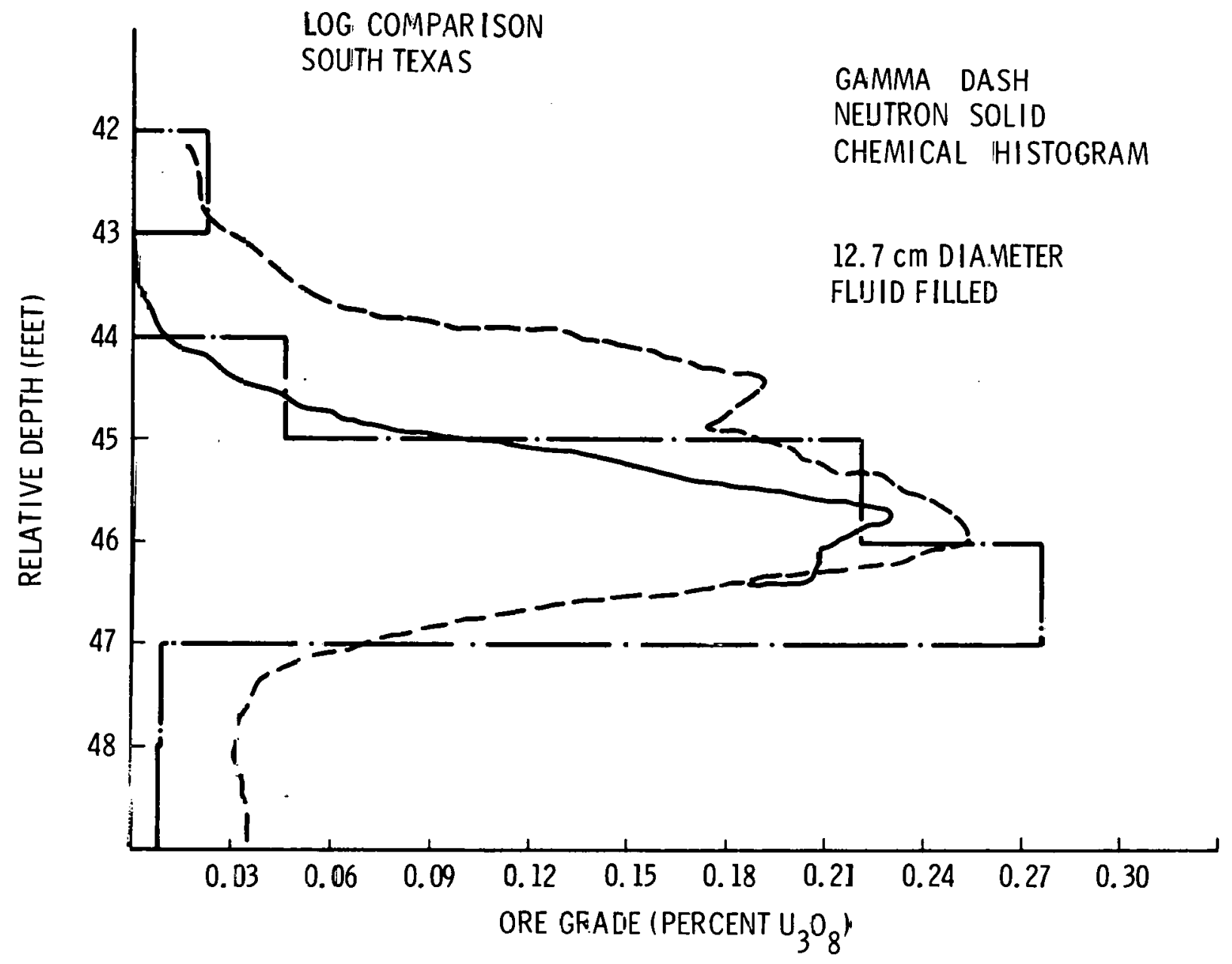

FIG. 28 


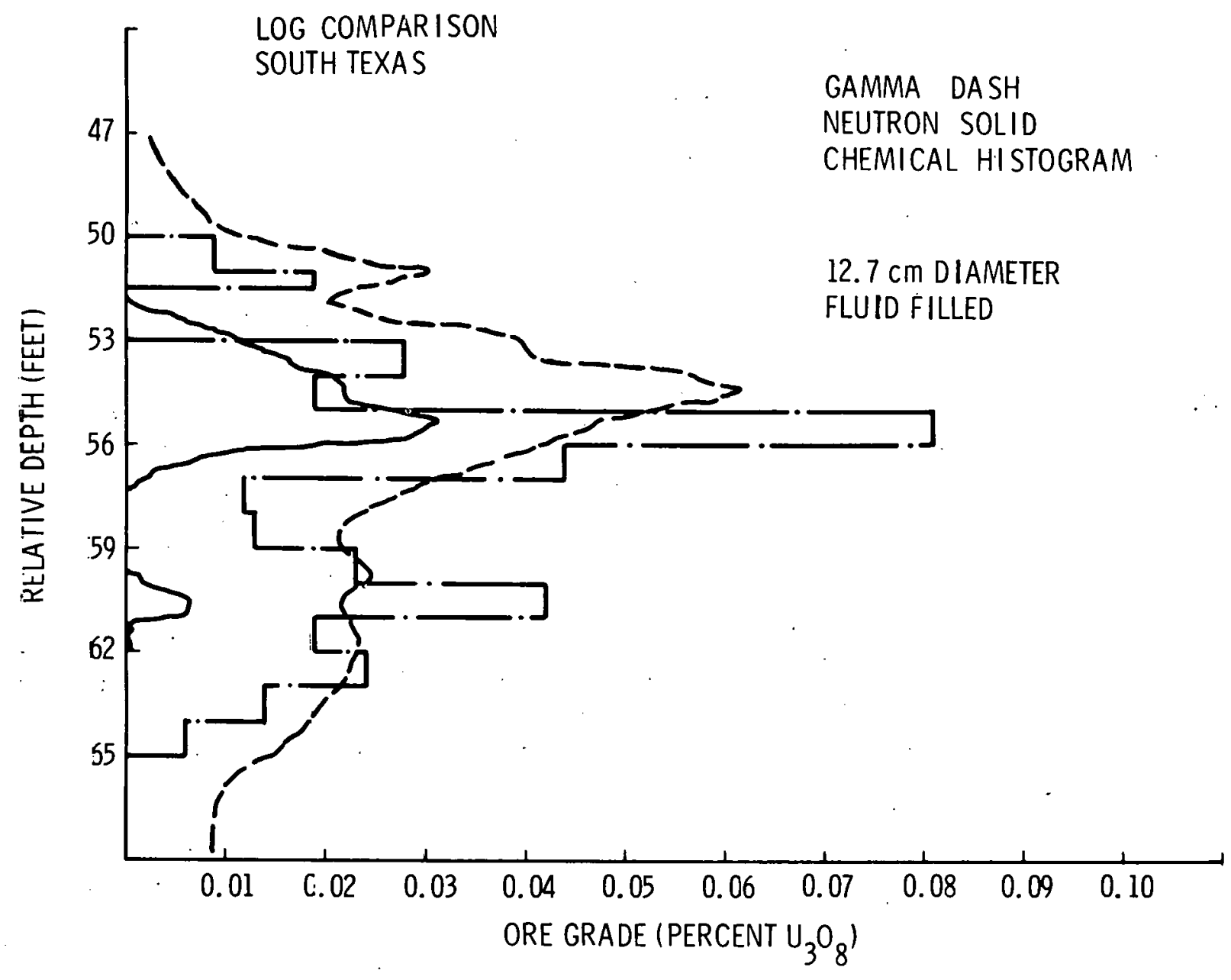

FIG. 29 


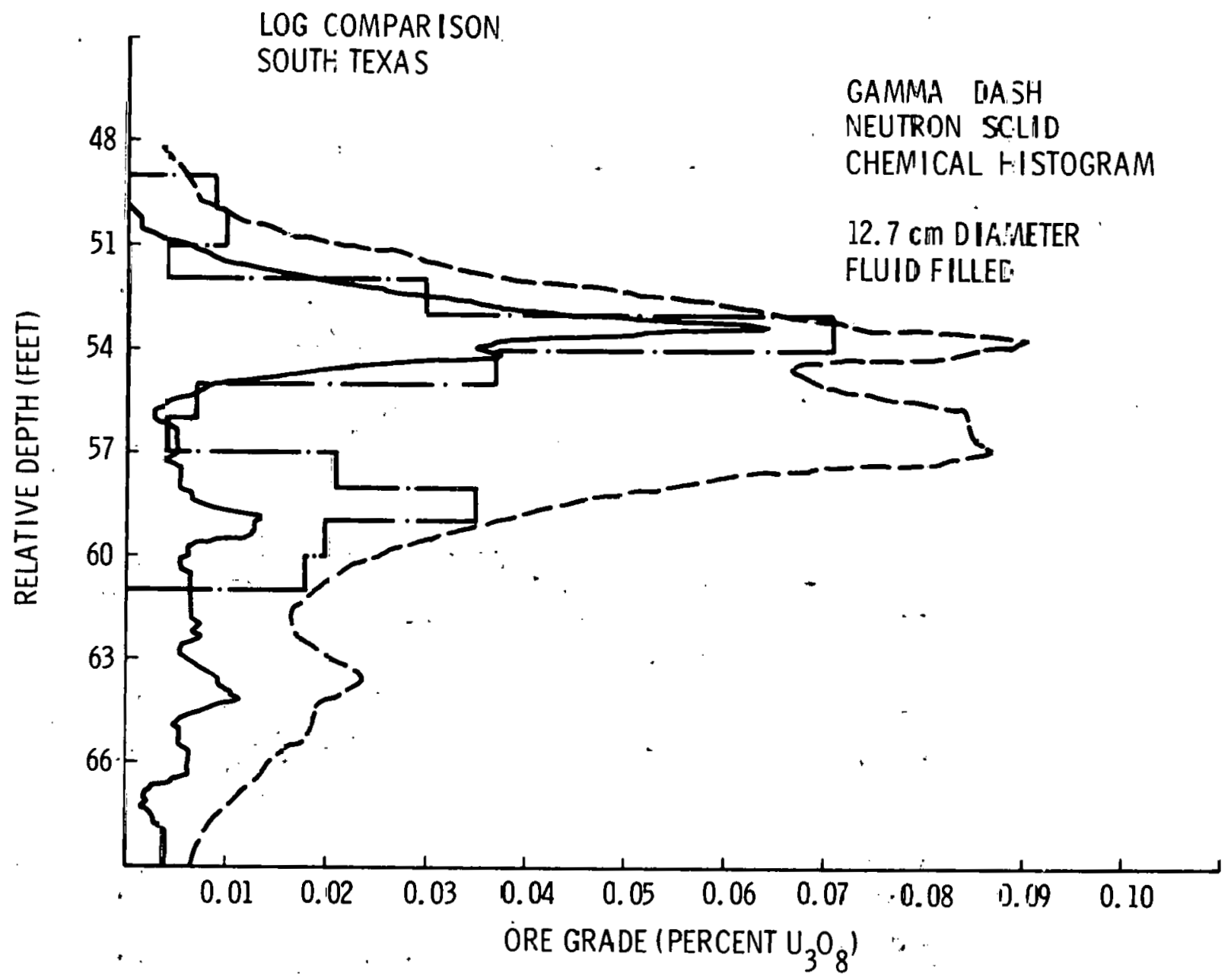

FIG. 30 


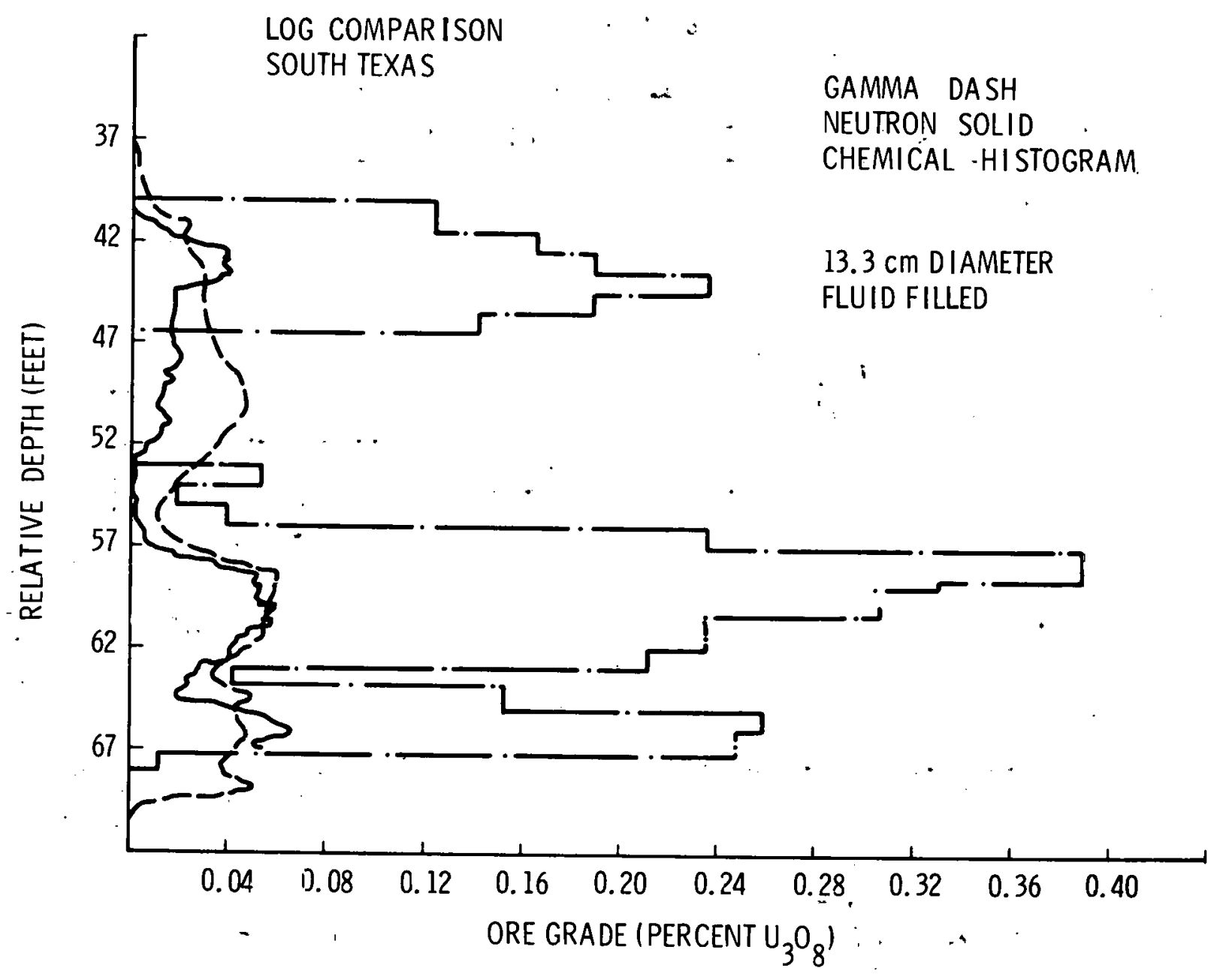

FIG. 31 


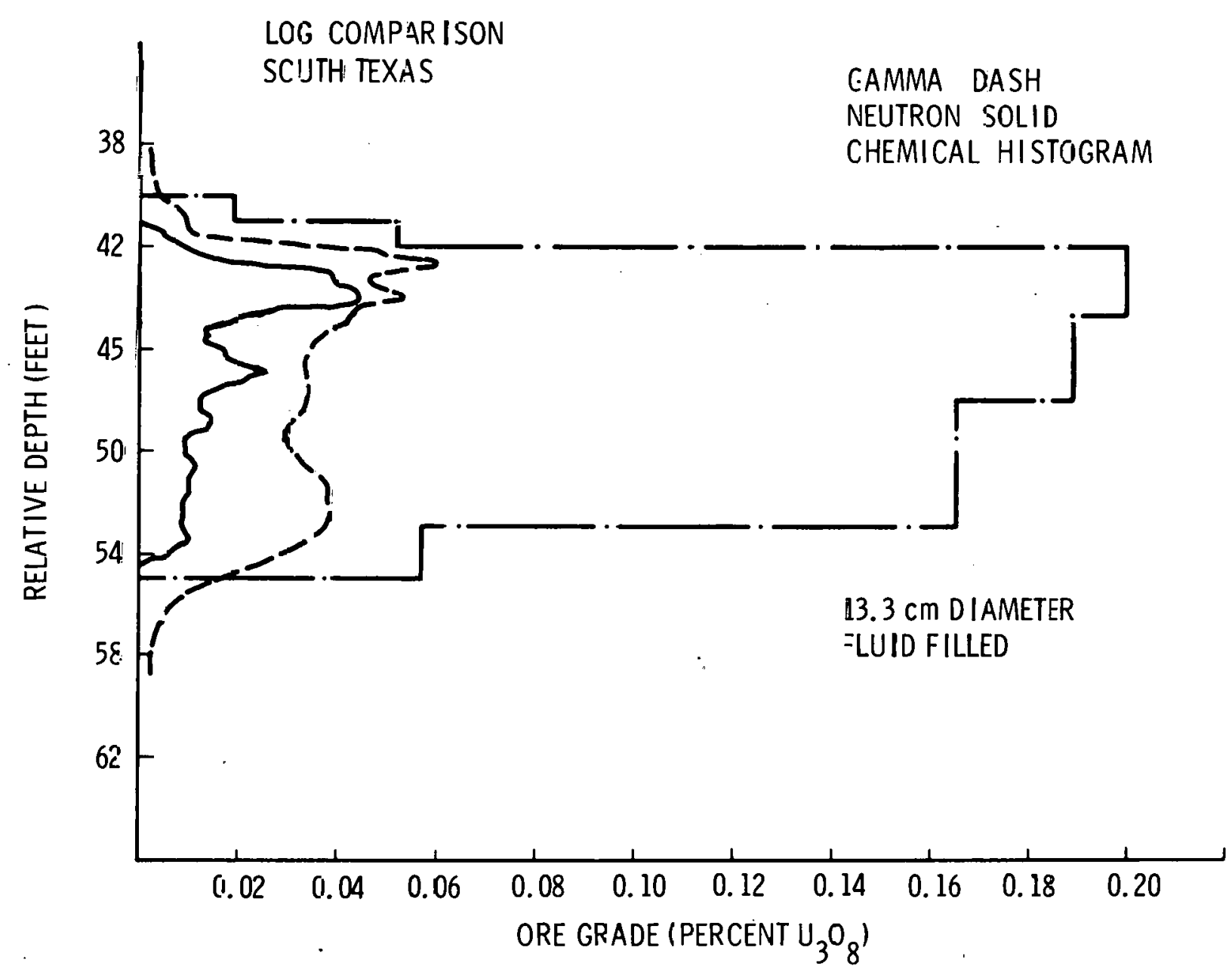

FIG. 32 


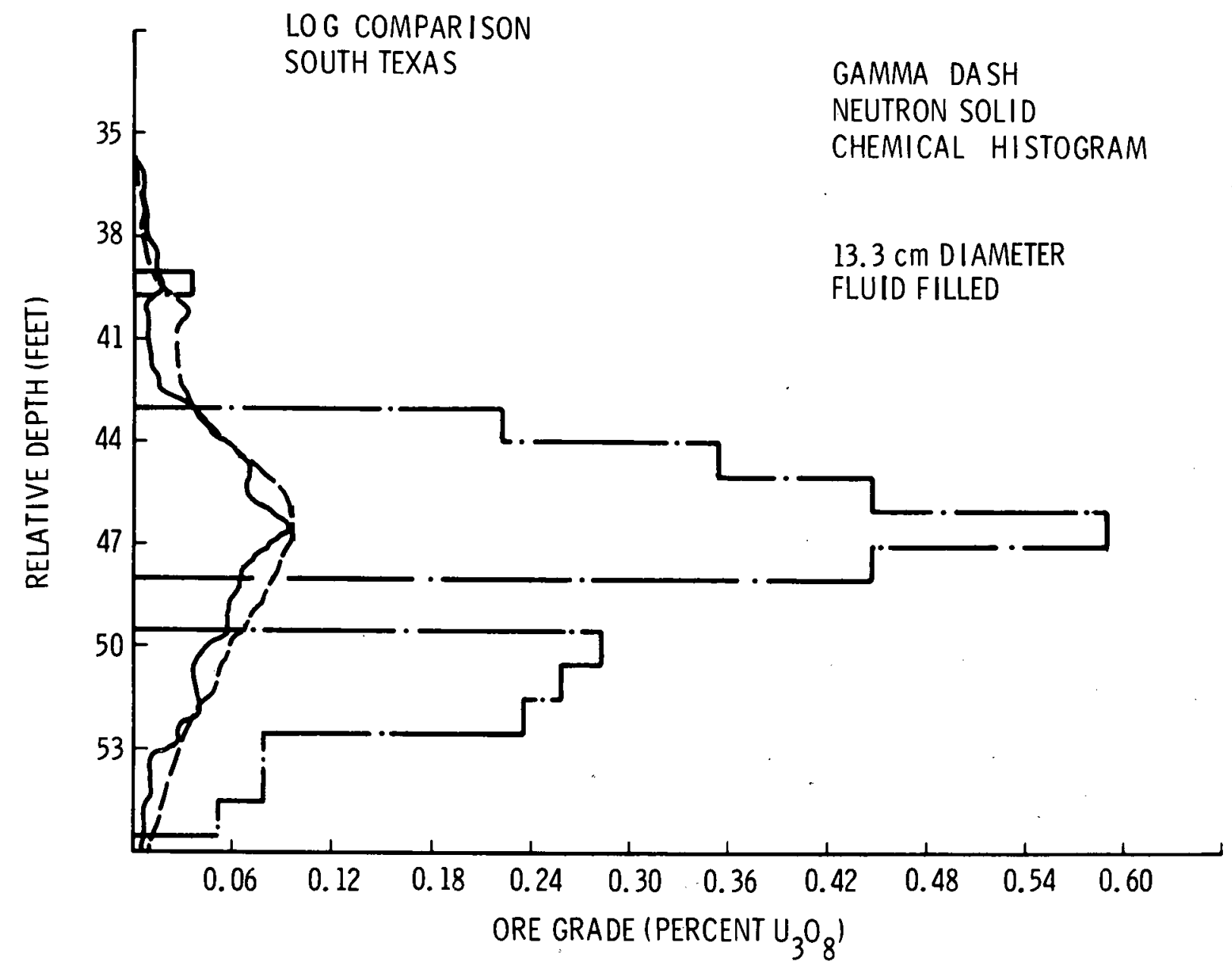

FIG. 33 
1. P. H. Dodd, "Neutron-Neutron Logging to Assay U-235 in Place," U.S.A.E.C. 1961, unpublished.

2. Jan A. Czubek, "Pulsed Neutron Method for Uranium Well Logging," Geophysics, Vo1. 37, No. $12-72$.

3. W. W. Givens, W. R. Mills, C. I. Dennis, and R. I. Caldwell, "Uranium Assay Logging Using a Pulsed $14 \mathrm{MeV}$ Neutron Source and Detection of Delayed Fission Neutrons," Geophysics, Vol. 41, No. 3, 468-489 (1976).

4. "Delayed Neutron Logging for Uranium with ${ }^{252} C_{f}$ " KERR-McGeE Corp. Californium - 252 Progress \#15, May 1973.

5. E. D. Cashwell, J. R. Neargaard, W. M. Taýlor, G. D. Turner," MCN: A Neutron Monte Carlo Code," LA 4751, January $19 \% 2$.

6. Nuclear Data: Model ND-LUU Muielchannel Analy 2 ei/Multiocalcr.

7. Pertec: Model 7840-75-25 Tape Drive.

8. I. J. Hall, D. D. Sheldon, Sandia Laboratories - internal communication.

9. F. D. Thibideau, "A Pulsed Neutron Generator for Logging," Technical Information Series, GEPP-249, February 1977. Available from NTIS.

10. Fluorinert FC-77, Trademark 3M Company.

11. Sorensen: Model DCR-600-1.5B.

12. Nuclear Data: Model 82-0371 MCS Buffer II.

13. Hewlett Packard: Model 9810A calculator, 9865A tape cassette, y864A a1g1tizer, and $9862 \mathrm{~A}$ plotter. 
Distribution

General Electric Company (3) Neutron Devices Department

P. O. Box 11508

St. Petersburg, FL 33733

2300

2350

2351

2351

2352

2353

2354

2354

2354

2354

2355

2355

2355

2355

2355

2355

2500

2540

2542

2542

2542

5131

5730

8266

3141

3151

L. D. Smith

J. C. Crawford

R. L. Dougherty

E. L. Jacobs

G. W. McClure

G. W. Perkins

B. E. Barnaby

F. M. Bacon

G. E. Boettcher

R. D. Volk

H. M. Bivens

R. W. Barnard (175)

R. E. Brooks

M. R. Heiser

D. H. Jensen

W. A. Stephenson

J. C. King

O. M. Stuetzer

$K$. R. Hessel

L. G. Rice

G. W. Smith (10)

P. C. Lysne

H. M. Staller

Attn: A. F. Veneruso

R. F. Traeger

E. A. Aas (2)

C. A. Pepmueller (5)

W. L. Garner (3)

For: ERDA/TIC (Unlimited Release)

3171-1 R. P. Campbell (25)
Dr. P. W. Levy

Brookhaven National Laboratory

Associated Universities

Upton, L.I., NY 11973 